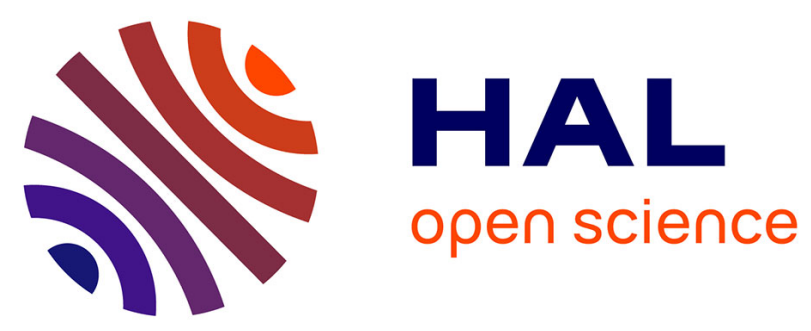

\title{
Neonatal susceptibility to meningitis results from the immaturity of epithelial barriers and gut microbiota
}

Laetitia Travier, Mariana Alonso, Alessio Andronico, Lukas Hafner, Olivier

Disson, Pierre-Marie Lledo, Simon Cauchemez, Marc Lecuit

\section{To cite this version:}

Laetitia Travier, Mariana Alonso, Alessio Andronico, Lukas Hafner, Olivier Disson, et al.. Neonatal susceptibility to meningitis results from the immaturity of epithelial barriers and gut microbiota. Cell Reports, 2021, 35 (13), pp.109319. 10.1016/j.celrep.2021.109319 . pasteur-03264312

\section{HAL Id: pasteur-03264312}

https://hal-pasteur.archives-ouvertes.fr/pasteur-03264312

Submitted on 31 Aug 2021

HAL is a multi-disciplinary open access archive for the deposit and dissemination of scientific research documents, whether they are published or not. The documents may come from teaching and research institutions in France or abroad, or from public or private research centers.
L'archive ouverte pluridisciplinaire HAL, est destinée au dépôt et à la diffusion de documents scientifiques de niveau recherche, publiés ou non, émanant des établissements d'enseignement et de recherche français ou étrangers, des laboratoires publics ou privés.

\section{(1) (1) $\$$}

Distributed under a Creative Commons Attribution - NonCommercial - NoDerivatives 44.0 


\section{Cell Reports}

\section{Neonatal susceptibility to meningitis results from the immaturity of epithelial barriers and gut microbiota}

\section{Graphical abstract}

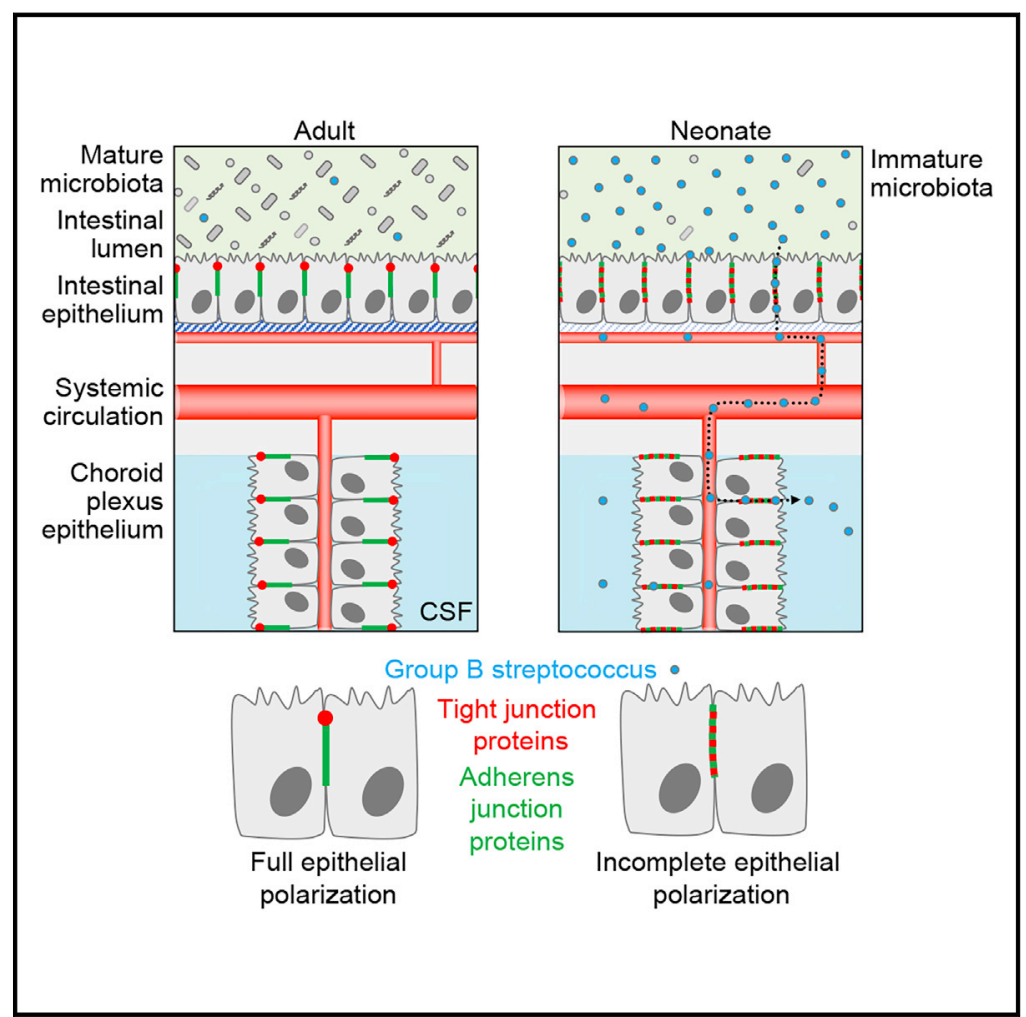

\section{Highlights}

- Age and microbiota immaturity account for neonatal susceptibility to GBS meningitis

- Immature microbiota favors GBS colonization of the neonatal gut lumen and bacteremia

- Neonatal intestine and choroid plexuses exhibit enhanced permissiveness to GBS

- Wnt signaling alters polarization of neonatal intestinal and choroid plexus epithelia

\section{Authors}

Laetitia Travier, Mariana Alonso, Alessio Andronico, ..., Pierre-Marie Lledo, Simon Cauchemez, Marc Lecuit

\section{Correspondence}

marc.lecuit@pasteur.fr

\section{In brief}

Travier et al. reveal the role of age and microbiota immaturity in neonatal susceptibility to GBS meningitis. Neonatal microbiota immaturity eases GBS gut colonization, translocation across the gut-vascular barrier, and bacteremia. Moreover, Wnt-associated incomplete polarization of the neonatal intestinal and choroid plexus epithelial barriers favors GBS translocation across these barriers. 


\title{
Article
}

\section{Neonatal susceptibility to meningitis results from the immaturity of epithelial barriers and gut microbiota}

\author{
Laetitia Travier, ${ }^{1,2}$ Mariana Alonso, ${ }^{3,4}$ Alessio Andronico, ${ }^{5,6}$ Lukas Hafner, ${ }^{1,2,7}$ Olivier Disson, ${ }^{1,2}$ Pierre-Marie Lledo, ${ }^{3,4}$ \\ Simon Cauchemez, ${ }^{5,6}$ and Marc Lecuit ${ }^{1,2,7,8,9,10, *}$ \\ ${ }^{1}$ Institut Pasteur, Biology of Infection Unit, Paris, France \\ 2Institut National de la Santé et de la Recherche Médicale U1117, Paris, France \\ ${ }^{3}$ Laboratory for Perception and Memory, Institut Pasteur, Paris, France \\ ${ }^{4}$ Centre National de la Recherche Scientifique, Unité Mixte de Recherche 3571, Paris, France \\ ${ }^{5}$ Mathematical Modelling of Infectious Diseases Unit, Institut Pasteur, Paris, France \\ ${ }^{6}$ Centre National de la Recherche Scientifique, Unité Mixte de Recherche 2000, Paris, France \\ 7 Université de Paris, Paris, France \\ ${ }^{8}$ National Reference Centre and WHO Collaborating Centre Listeria, Institut Pasteur, Paris, France \\ ${ }^{9}$ Necker-Enfants Malades University Hospital, Department of Infectious Diseases and Tropical Medicine, APHP, Institut Imagine, Paris, \\ France \\ 10 Lead contact \\ *Correspondence: marc.lecuit@pasteur.fr \\ https://doi.org/10.1016/j.celrep.2021.109319
}

\section{SUMMARY}

Neonates are highly susceptible to bacterial meningitis as compared to children and adults. Group B streptococcus (GBS) is a major cause of neonatal meningitis. Neonatal meningitis can result from GBS intestinal colonization and translocation across the intestinal barrier (IB). Here, we show that the immaturity of the neonatal intestinal microbiota leads to low resistance to GBS intestinal colonization and permissiveness of the gut-vascular barrier. Moreover, the age-dependent but microbiota-independent Wnt activity in intestinal and choroid plexus (CP) epithelia results in a lower degree of cell-cell junctions' polarization, which favors bacterial translocation. This study thus reveals that neonatal susceptibility to GBS meningitis results from the age-dependent immaturity of the intestinal microbiota and developmental pathways associated with neonatal tissue growth, which both concur to GBS gut colonization, systemic dissemination, and neuroinvasion. Whereas the activation of developmental pathways is intrinsic to neonates, interventions aimed at maturing the microbiota may help prevent neonatal meningitis.

\section{INTRODUCTION}

Bacterial pathogens that reach the brain via the systemic circulation cross the blood-brain barrier (BBB) via (1) brain microvessels, also called the BBB per se; and/or (2) the blood-meningeal barrier (BMB); and/or (3) the blood-cerebrospinal fluid (CSF) barrier, which is located at the choroid plexuses (CP) level (Disson and Lecuit, 2012; Lemichez et al., 2010; Saunders et al., 2018). Neonates are more susceptible than adults to bacterial meningitis, and meningitis is a frequent complication of bacteremia in neonates (Kim, 2010). Moreover, the etiologies of bacterial meningitis vary with age: whereas Neisseria meningitidis and Streptococcus pneumoniae are the main causes of meningitis in children and adults, in whom they colonize the nasopharynx, neonatal meningitis is almost exclusively caused by Streptococcus agalactiae (group B streptococcus [GBS]) and Escherichia coli, which are intestinal commensals (Badri et al., 1977; Edmond et al., 2012; Hansen et al., 2004; Tazi et al., 2019). There are two types of neonatal infections: early-onset disease, which occurs in the first week of life, and late-onset disease (LOD) that develops from 7 days to 3 months after birth. In total, $68 \%$ of GBS neonatal meningitis cases are LOD (Romain et al., 2018). Whereas GBS-associated early-onset disease results from GBS inhalation during parturition, GBS-associated LOD is thought to require pre- or post-delivery mother-to-child GBS transmission and subsequent intestinal seeding (Bingen et al., 1992; Romain et al., 2018; Tazi et al., 2019). The majority of LODs are due to the capsular serotype III GBS hypervirulent clonal complex CC17 (Tazi et al., 2019, 2010). Its overrepresentation in LOD cases seems to result from its enhanced capacity to colonize the neonatal gastrointestinal tract and translocate across host barriers (Tazi et al., 2019, 2010).

Neonatal microbiota composition derives from the early opportunistic colonization by the first bacterial species of maternal, food, and environmental origins to which neonates are exposed. The intestinal microbiota matures with age in response to changes and diversification of diet and environmental bacterial sources, until the age of 2 or 3 years in humans 

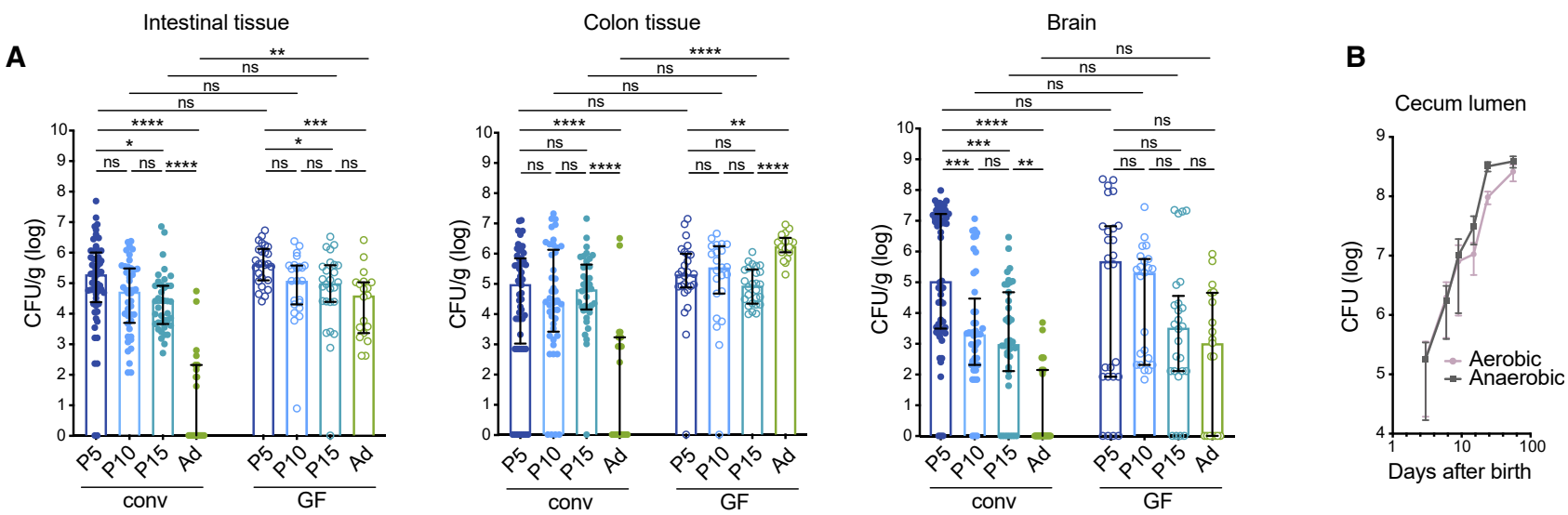

C

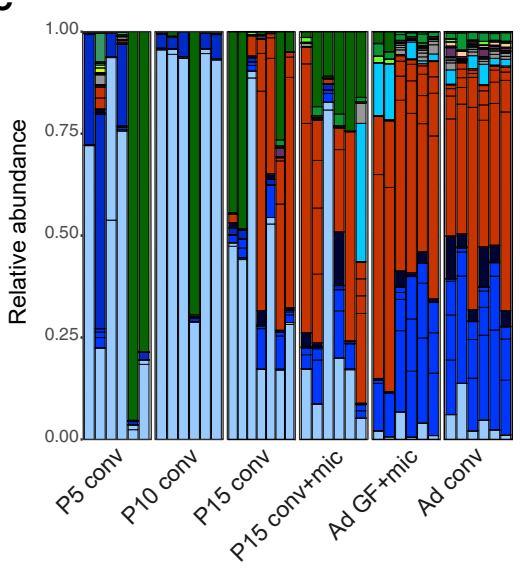

Proteobacteria, Enterobacteriales Proteobacteria, Desulfovibrional Proteobacteria, Sphingomonadales Proteobacteria, Rhizobiales Proteobacteria Rhodobacterales Proteobacteria, Burkholderiales Proteobacteria, Pseudomonadales Proteobacteria, Rhodospirillales Proteobateria, Oceanospirillales Proteobacteria, Caulobacterales

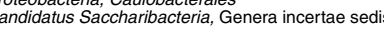
Actinobacteria, Coriobacteriales Actinobacteria, Bifidobacteriales Verrucomicrobia, Verrucomicrobiales Bacteroidetes, Bacteroidales Bacteroidetes, Flavobacteriales Bacteroidetes, Sphingobacterial Firmicutes, Erysipelotrichales Firmicutes, Clostridia Firmicutes, Lactobacillales
D

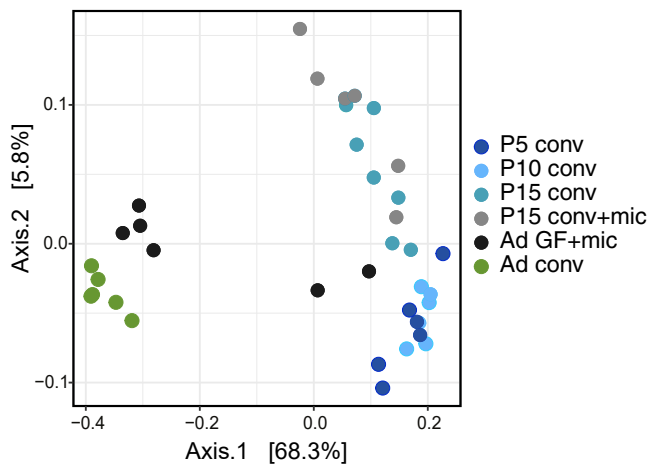

E
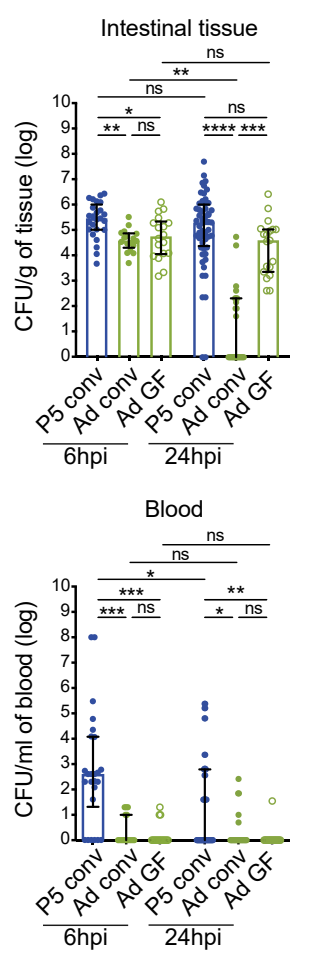
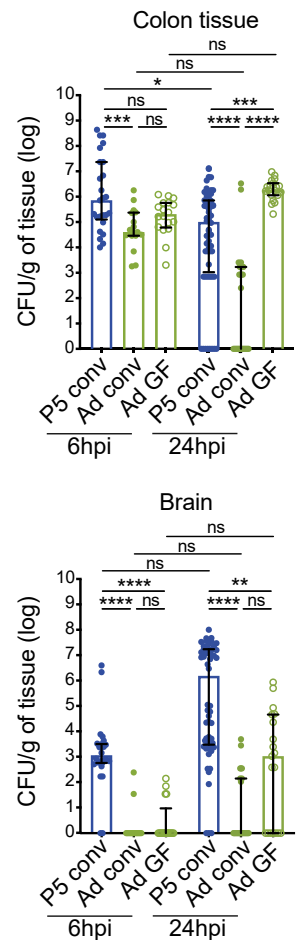

F
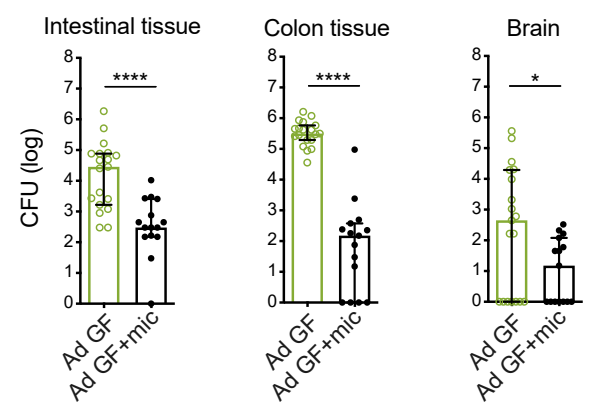

G

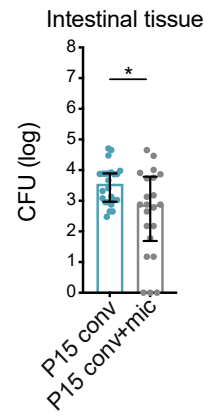

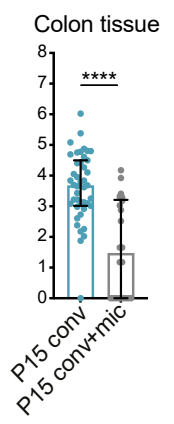

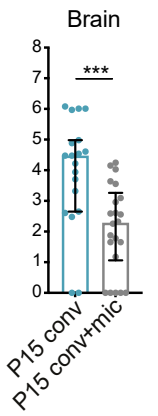


(Jain and Walker, 2015; Salazar et al., 2014). The gut microbiota helps adsorb and modify nutrients (Sonnenburg and Bäckhed, 2016) and mediate resistance against intestinal lumen colonization by orally acquired bacteria (Buffie and Pamer, 2013; Keith and Pamer, 2019). The neonatal microbiota is also involved in intestinal homeostasis (Belkaid and Hand, 2014; Hooper et al., 2001; Johansson et al., 2015) and maturation of the immune system (Deshmukh et al., 2014; Gensollen et al., 2016; Khosravi et al., 2014). Moreover, the maternal microbiota has been reported to regulate the tightness of fetal brain microvessels' junctions (Braniste et al., 2014).

Here, we aimed at understanding the reasons for neonatal susceptibility to GBS LOD meningitis. We show that neonatal susceptibility to GBS neonatal meningitis results from a succession of age-dependent, microbiota-dependent, and microbiotaindependent factors. We demonstrate that neonatal intestinal microbiota immaturity is associated with decreased resistance to GBS colonization, which favors translocation across the gut barrier. We also identify that neonatal microbiota immaturity is associated with leakiness of the gut vascular barrier (GVB) and higher systemic bacterial load. Finally, we reveal a key role for the microbiota-independent but age-dependent Wnt activity in preventing the full polarization of cell-cell epithelial junctions, which favors bacterial translocation across the intestinal (IB) and CP barriers.

\section{RESULTS}

Impact of age and the intestinal microbiota on host susceptibility to bacterial meningitis

To investigate the impact of age on host susceptibility to GBS LOD-associated meningitis, conventional mice of increasing age were orally inoculated with GBS. In line with epidemiological observations in human, GBS burden $24 \mathrm{~h}$ post-oral inoculation significantly decreased according to age in intestinal tissue, colon, and brain (Figure 1A, left panels of each graph).

Since the intestinal microbiota maturates with age, we assessed the relation between the age-dependent microbiota maturation and age per se on host susceptibility to GBS infection by comparing germ-free (GF) and conventional mice of different ages. We first confirmed that the number of cultivable bacteria in the gut lumen increases with age (Figure 1B) and that microbiota composition changes from Enterobacteriales and Lactobacil- lales in P5 and P10 to Clostridiales and Bacteroidales in P15 and adult as dominant orders, associated with an increase in microbiota diversity (Figures 1C, 1D, S1A, S1B, and S2). This illustrates, as previously reported, the immaturity of the neonatal gut microbiota (Jain and Walker, 2015; Salazar et al., 2014).

While the susceptibility to GBS, as assessed by bacterial burden in tissues $24 \mathrm{~h}$ after oral inoculation, was microbiota dependent in adult mice, there was no difference at P5 between GF and conventional mice (Figure 1A), highlighting the immaturity of the microbiota at P5 (Figure S1A). The bacterial burden increased in the brain of conventional P5 and GF adult mice between 6 and $24 \mathrm{~h}$ post-inoculation, whereas it decreased in conventional adult mice (Figure 1E). In line with this result, transfer of gut luminal content from conventional to GF adult mice and to conventional neonatal mice reduced GBS bacterial burden in gut tissues and the brain (Figures 1F, 1G, and S1C), demonstrating that mature microbiota protects mice from GBS meningitis (Figures 1C, 1D, S1A-S1E, and S2).

Neonatal susceptibility to GBS was, however, not purely microbiota dependent: $24 \mathrm{~h}$ after oral-inoculation in GF mice, bacterial burden was lower in adults compared to neonates, both in the intestinal tissue and in the brain (Figure 1A, right panels of each graph). This suggests that neonatal susceptibility to GBS also depends on age per se, independent of microbiota. Consistently, as early as $6 \mathrm{~h}$ after oral inoculation, bacterial burden was significantly higher in conventional P5 intestinal tissue, blood, and brain than in conventional and GF adult mice (Figure 1E), showing that early invasion of the intestinal tissue by GBS strongly depends on age per se.

Together, these results show that neonatal susceptibility to GBS infection strongly depends both on gut microbiota maturity and age per se. To determine how age and microbiota each modulate susceptibility to GBS meningitis, we analyzed step by step the infection process by characterizing GBS (1) colonization of the gut lumen, (2) crossing of the IB, (3) bacteremia control, and (4) crossing of the BBB.

\section{Intestinal colonization resistance and GBS gut tissue invasion}

In order to decipher the mechanisms of microbiota impact on neonatal susceptibility to meningitis, we first assessed the resistance to GBS gut colonization of neonatal versus adult mice. Five days post-oral inoculation, GBS load in gut lumen was higher in

Figure 1. Age and intestinal microbiota immaturity account for neonatal susceptibility to meningitis

(A) GBS count/g of gut tissues and brain of conventional P5 ( $n=56)$, P10 ( $n=44)$, P15 ( $n=39)$, and adult ( $n=26)($ left panels) and GF P5 ( $n=26)$, P10 ( $n=23)$, P15 $(n=26)$, and adult $(n=19)$ (right panels) mice $24 \mathrm{~h}$ post-oral inoculation with $5 \times 10^{8} \mathrm{GBS} / \mathrm{g}$ of mice.

(B) Commensals count in the gut lumen of conventional P3 ( $=5)$, P6 ( $=3)$, P9 ( $=3)$, P15 $(n=4)$, P24 ( $n=3)$, and adult ( $n=3)$ mice.

(C) Relative abundance of phylum- and order-level commensals obtained from 16S rDNA sequencing of the gut contents of conventional P5 $(n=6)$, P10 $(n=6)$, P15 $(n=7)$, and adult $(n=6)$ mice and of GF adult $(n=6)$ and P15 conventional $(n=6)$ mice that were orally inoculated with gut luminal content of conventional adult mice (Ad GF+mic and P15 conv+mic, respectively).

(D) Beta diversity between mice groups from (C) shown as principal coordinate analysis of Bray-Curtis distances between samples.

(E) GBS count/g of organ or per ml of blood in conventional P5 and adult and GF adult mice $6 \mathrm{~h}$ ( $\mathrm{n}=26,17$, and 18 , respectively) and $24 \mathrm{~h}$ post-oral inoculation with $5 \times 10^{8} \mathrm{GBS} / \mathrm{g}$ of mice (same data as A for 24 -h time point).

(F) GBS count in GF adult mice $24 \mathrm{~h}$ post-oral inoculation with $10^{10} \mathrm{GBS}$. Two weeks before inoculation, mice were orally inoculated with PBS (Ad GF, $\mathrm{n}=19$ ) or with gut luminal content isolated from conventional adult mice (Ad GF+mic, $n=15$ ).

(G) GBS count in P15 conventional mice 24 h post-oral inoculation with $3.10^{9}$ GBS. Before oral inoculation, mice were inoculated at P3, P6, and P9 with PBS (P15 conv, $n=19$ ) or with gut luminal content of conventional adult mice (P15 conv+mic, $n=22)$ (see Figure $S 1 C)$.

Statistical tests: Kruskal-Wallis (A and E), Mann-Whitney (F and G). Conventional, conv; adult, Ad. Representation: median \pm interquartile range (IQR). 
A
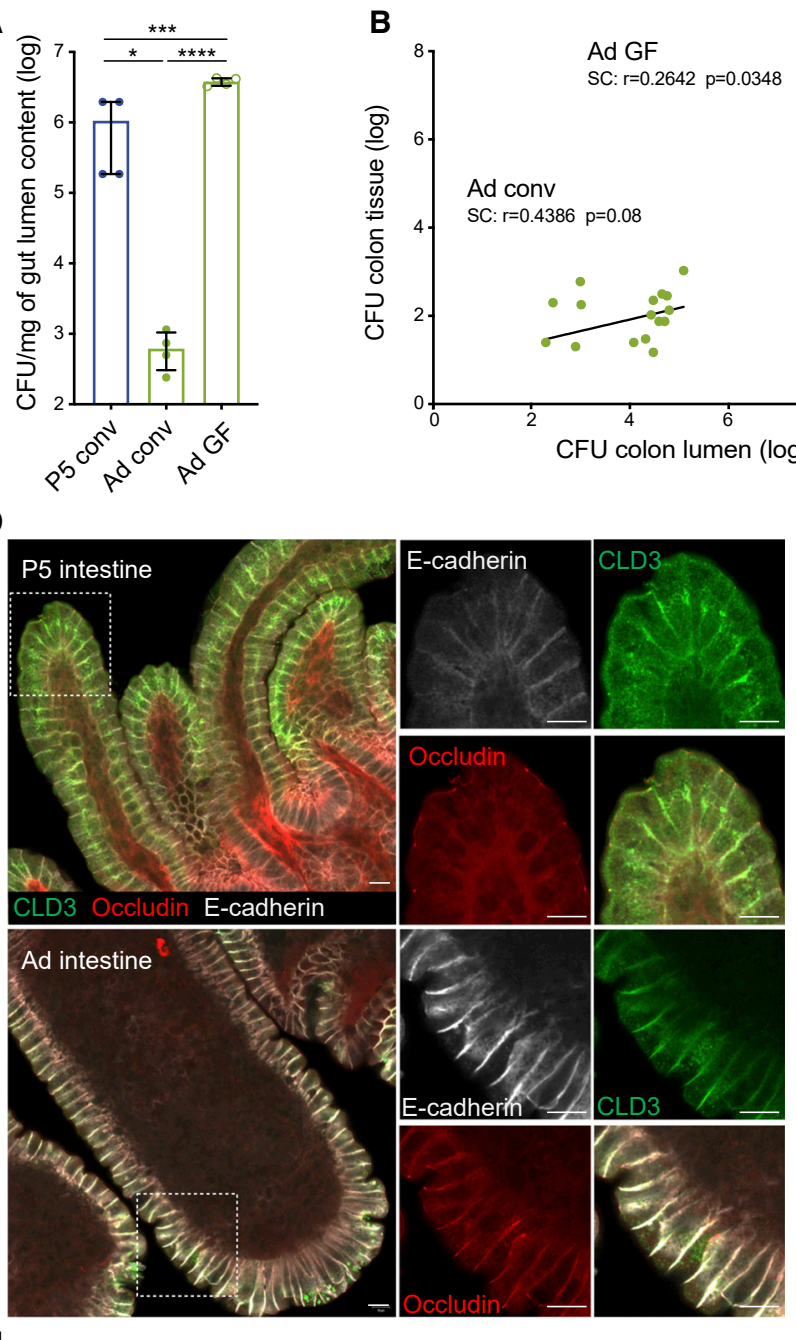

H

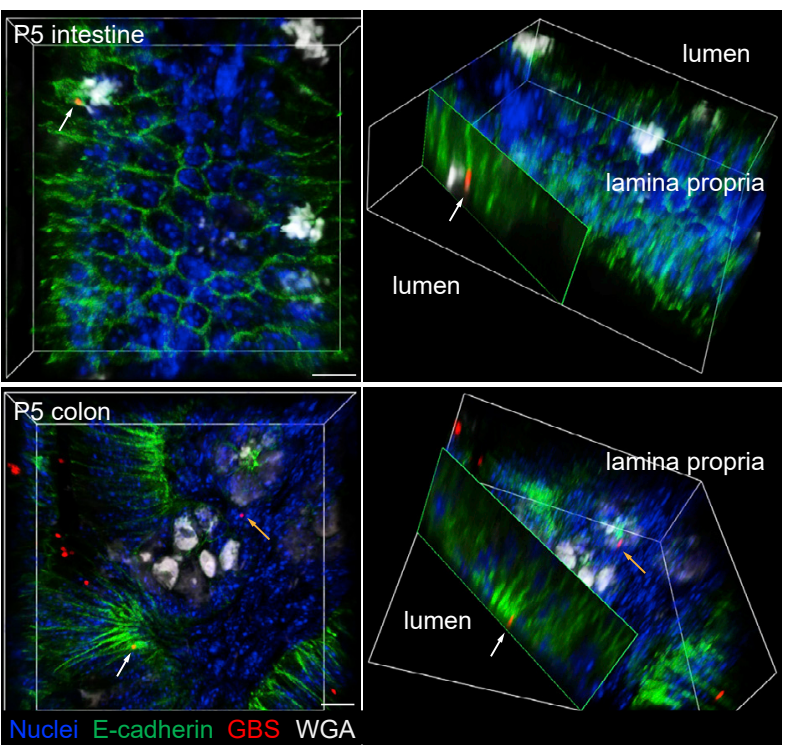

B

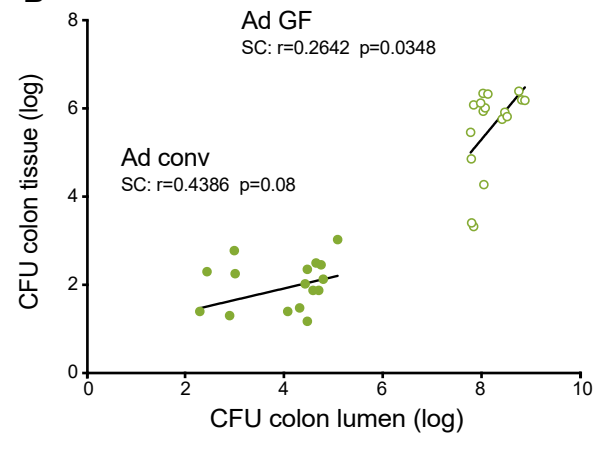

C

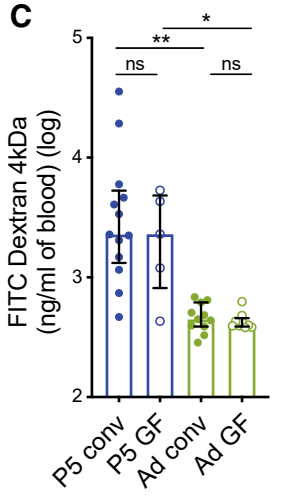

E

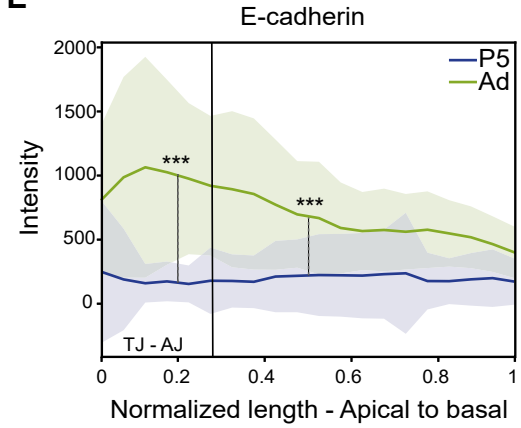

$\mathbf{F}$

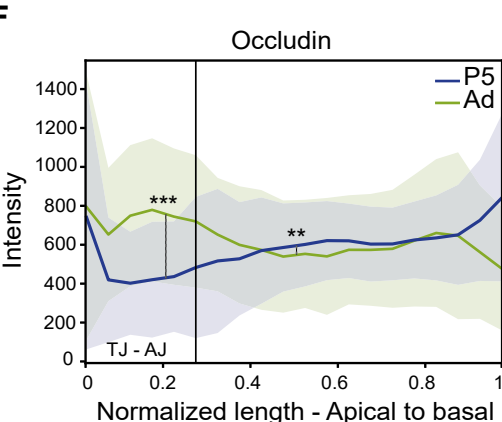

G

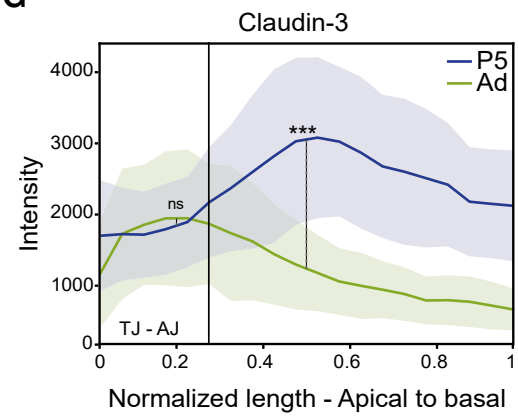


conventional P5 mice than in conventional adult mice but was lower than the bacterial load in orally inoculated adult GF mice (Figure 2A), suggesting that the developing microbiota in P5 mice already confers some intestinal colonization protection compared to GF mice. Accordingly, $24 \mathrm{~h}$ post-oral inoculation, GBS load upon gentamicin treatment, which kills extra-tissular GBS (Tazi et al., 2010), was higher in the gut tissue of adult inoculated GF mice than conventional mice (Figures 1A, 1E, and 2B). Of note, in GF adult mice, GBS colon infection positively correlated with GBS luminal content load (Figure 2B), in contrast to the intestine (Figure S3A), although one cannot exclude that a fraction of luminal GBS survives to gentamicin and that tissue colon burden can be slightly overstated. Together, these results indicate that mature microbiota mediates colonization resistance to GBS and tend to strengthen gut barrier function at the colon level, thereby further limiting GBS invasion of gut tissue.

\section{Age-dependent permissiveness of the neonatal IB and} polarization of intestinal epithelial junctions

We next asked whether the IB is more permissive in neonates than in adults, independently of microbiota-driven colonization resistance. This hypothesis is supported by our observation that infection of the gut tissues is influenced by age in a microbiota-independent manner (Figures $1 \mathrm{~A}$ and $1 \mathrm{E}$ ), which may reflect a higher permissiveness of the IB in younger animals. Four hours post-oral inoculation of fluorescein isothiocyanate (FITC)-Dextran-4kDa (Al Nabhani et al., 2019), dye concentration was higher in GF and conventional P5 mice blood than in adult, indicating that IB is indeed more permissive in neonatal than adult mice (Figure 2C). We therefore compared intestinal epithelial cell-cell junctions in neonatal and adult mice. The quantification of the transcript level of genes encoding adherens junction (AJ) protein E-cadherin and tight junction (TJ) proteins Occludin and Claudin-3 in the whole intestinal tissue showed no significant difference for Cdh1 (E-cadherin), a lower level for Cldn3 (claudin-3), and a higher level for Ocln (occludin) in P5 compared to adult mice (Figure S3B). However, analysis of signal intensity and location of junction proteins along small intestine epithelial junctions showed that E-cadherin signal is less abundant and apically concentrated in P5 junctions than in adult (Figures 2D and 2E). Indeed, E-cadherin signal did not peak at AJs in P5 mice as it does in adult mice (Figure 2E). In addition, occludin and claudin-3 signals in P5 junctions were not shifted toward the apical region of the epithelial cells, in contrast to mature TJs of adult mice (Figures 2D, 2F, and 2G). These results show that expression and polarization of $\mathrm{AJ}$ and $\mathrm{TJ}$ proteins are weaker in the neonatal intestinal epithelium than in adults, in agreement with the lower barrier function of the neonatal IB as compared to that of adult mice (Figure 2C). Of note, E-cadherin, occludin, and claudin-3 signals polarized at AJs and TJs in GF adult mice as in conventional adult mice, despite lower occludin and higher claudin-3 intensity signals (Figures S3C-S3F), confirming that the incomplete polarization of the neonatal intestinal epithelium is age-dependent but microbiota independent. In P5 mice orally inoculated with GBS for $6 \mathrm{~h}$, we detected GBS embedded in epithelial junctions and within the lamina propria of the intestine (Figures $2 \mathrm{H}$ and S3G). Of note, the same observation was made in the colon tissue (Figure $2 \mathrm{H}$ ) in which quantification of the transcript level of genes coding for AJ and TJ proteins showed lower levels of Cdh1, Ocln, and Cldn3 expression in P5, compared to adult mice (Figure S3H). These results suggest that GBS takes advantage of the lower tightness of epithelial cell-cell junctions in neonatal gut tissue to cross the neonatal IB in a paracellular way, as described in in vitro cultured epithelial cells (Soriani et al., 2006).

\section{Wnt pathway activity in the neonatal intestinal epithelium}

E-cadherin is stabilized at the cell surface via the interaction of its cytoplasmic domain with $\beta$-catenin, which connects it to the actin cytoskeleton via $\alpha$-catenin. Phosphorylation of $\beta$-catenin disconnects it from $E$-cadherin, which can thus be recycled. $\beta$-catenin released in the cytosol accumulates upon activation of the Wnt pathway, leading to a downregulation of E-cadherin expression (Bhatt et al., 2013; Coopman and Djiane, 2016). Given the observed difference in E-cadherin levels between neonatal and adult intestinal epithelial cell-cell junctions (Figures 2D and 2E), we investigated the expression level and localization of $\beta$-catenin in the neonatal intestine as compared to that of adult mice, knowing that the activation of the Wnt canonical pathway blocks $\beta$-catenin proteasomal degradation and favors the destabilization of AJs and E-cadherin recycling. $\beta$-catenin signal was found to be higher in P5 epithelium than in adult, both at cell-cell junctions and in the cytoplasm (Figures 3A-3C). Accordingly, E-cadherin signal was lower and more dispersed at the P5 junctions and brighter in the epithelial cytoplasm compared to that of adults

Figure 2. Lower resistance to intestinal colonization and higher permissiveness of the IB in neonates

(A) GBS count/g of gut luminal content of conventional P5 and adult and GF adult mice 5 days post-oral inoculation with $10^{7}$ GBS/g of mice ( $\mathrm{n}=4$ in each group). (B) Spearman correlation (SC) between GBS count in the colon lumen and tissue of conventional and GF adult mice ( $\mathrm{n}=17$ ) $24 \mathrm{~h}$ post-oral inoculation with $10^{10}$ GBS.

(C) FITC-Dextran 4kDa concentration in blood of conventional P5 $(n=13)$ and adult $(n=11)$ and GF P5 ( $n=5)$ and adult $(n=9) 4$ h post-oral inoculation with 0.5 mg/ $\mathrm{g}$ of mice.

(D) Confocal imaging of 0.4- $\mu$ m-thick sections of conventional P5 and adult small intestine. Sections were stained for claudin-3 (CLD3), occludin, and E-cadherin. Scale bars, $10 \mu \mathrm{m}$.

$(E-G)$ E-cadherin $(E)$, occludin $(F)$, and claudin-3 $(G)$ intensity signal from $(D)$, measured along the length-normalized epithelial junctions, from the apical to the basal side ( $n=85$ in P5, $n=60$ in adult). Tight (TJs) and adherens junctions (AJs) are located in the left part of the vertical line. Signal distribution was compared at length junction units 0.2 and 0.5 (vertical dotted line).

(H) 3D reconstruction and planal clipping view (yz view) of confocal imaging of $150-\mu \mathrm{m}$-thick sections of P5 gut tissues $6 \mathrm{~h}$ post-oral inoculation with $10^{9} \mathrm{GBS}$. Sections were stained for nuclei, E-cadherin, GBS, and mucus (wheat germ agglutinin [WGA]). White arrows, GBS within junctions; orange arrow, GBS within lamina propria. Scale bar, $10 \mu \mathrm{m}$.

Statistical tests: one-way ANOVA (A), SC (B), Kruskal-Wallis (C), Mood (E-G). Median \pm IQR. 
A

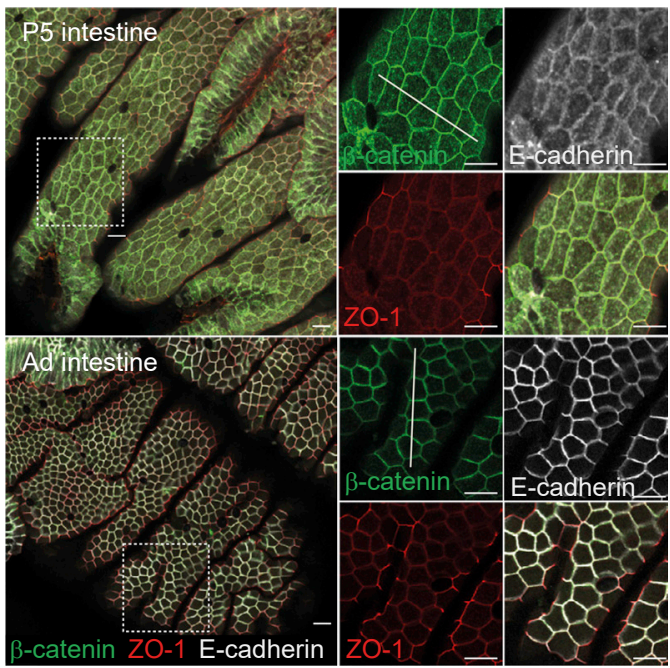

C
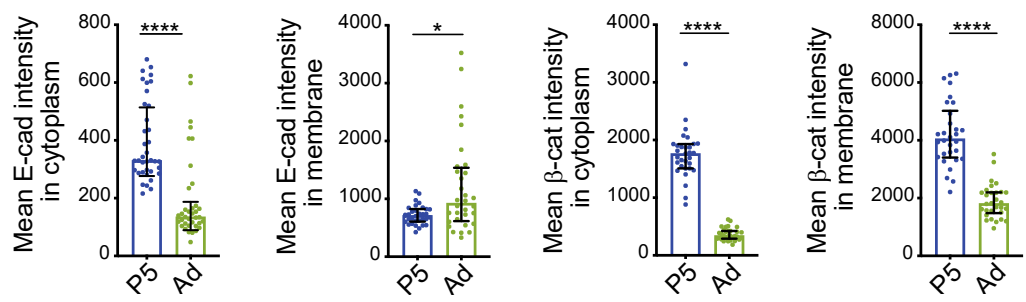
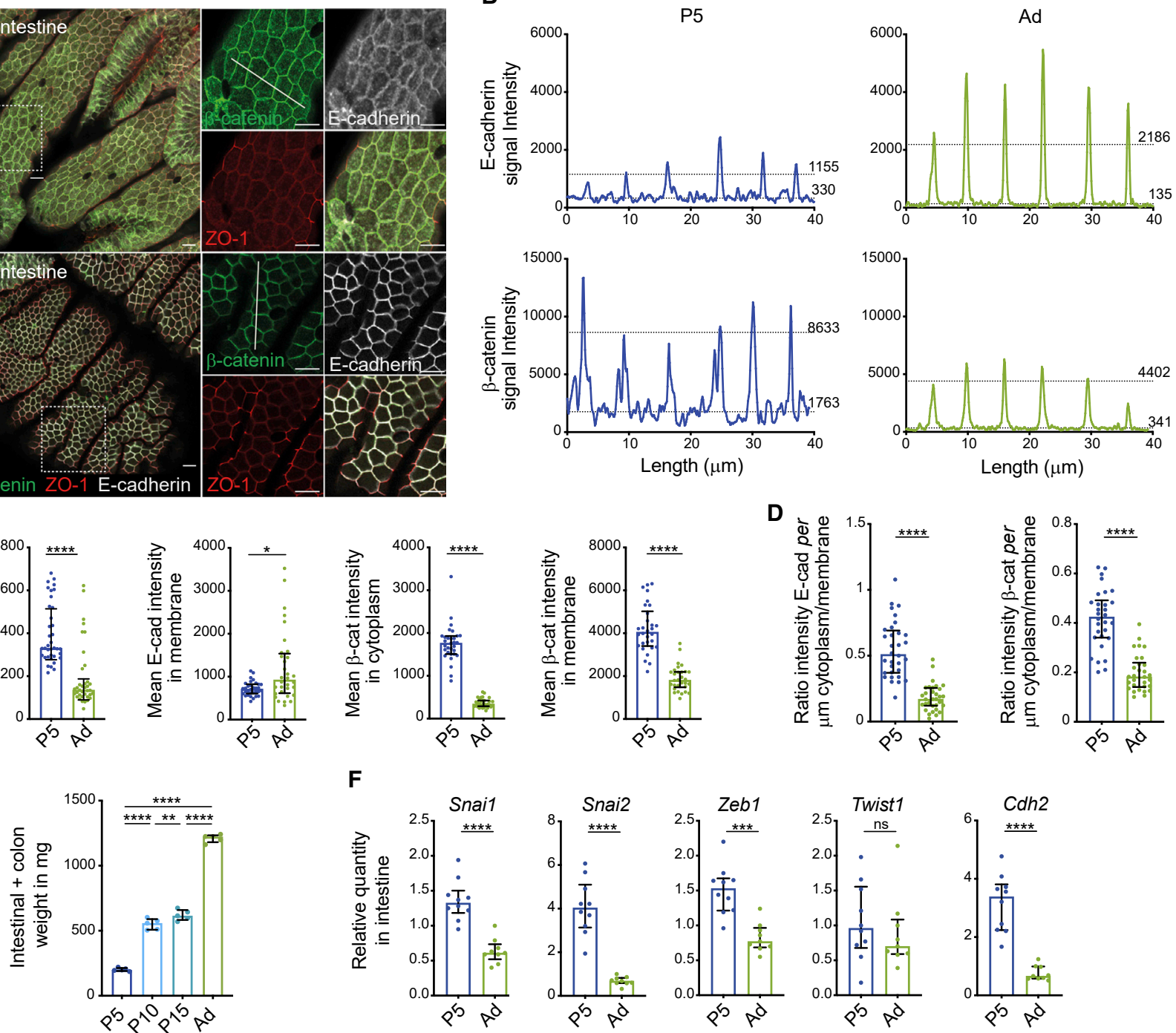

F
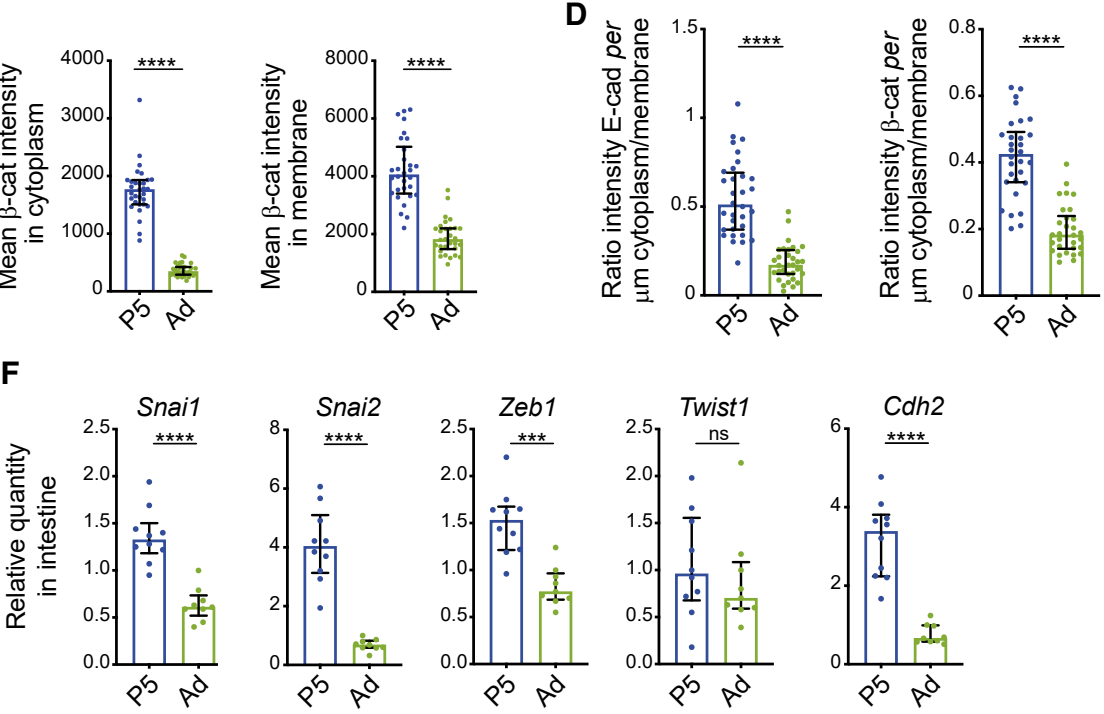

Figure 3. Higher Wnt activity in the neonatal IB

(A) Confocal imaging of $0.4-\mu \mathrm{m}$-thick sections of conventional P5 and adult small intestine. Sections were stained for $\beta$-catenin, ZO-1, and E-cadherin. Cellular membranous and cytoplasmic signal intensity for $\beta$-catenin and E-cadherin was analyzed along drawn white line. Scale bars, $10 \mu \mathrm{m}$.

(B) Representative $\beta$-catenin and E-cadherin signal intensity from (A) measured along the drawn white line. The lower and upper dotted lines represent the median intensity signal in cytoplasm and in membranes, respectively, and respective values are written on the right of each line.

$(C$ and $D)$ Mean $(C)$ and ratio (D) of $\beta$-catenin ( $\beta$-cat) and E-cadherin (E-cad) intensity signal in cytoplasm and membranes of intestinal epithelial cells stained in (A) $(\mathrm{n}=32)$.

(E) Weight of the small intestine and colon in P5, P10, P15, and adult mice $(n=5)$.

(F) Relative expression of Snai1 (Snail1), Snai2 (Slug), Zeb1 (Zeb1), Twist1 (Twist1), and Cdh2 (N-cadherin) in the intestinal tissue of conventional P5 ( $\mathrm{n}=10$ ) and adult $(n=9)$ mice.

Statistical tests: Mann-Whitney (C, D, and F), one-way ANOVA (E). Median \pm IQR.

(Figures 3A-3C). Ratios of cytoplasmic versus junction-associated $\mathrm{E}$-cadherin and $\beta$-catenin signals indicated that the proportion of both cytoplasmic E-cadherin and $\beta$-catenin over that associated to junctions is higher in neonates than in adults (Figure 3D) and is not influenced by microbiota (Figures S4A and S4B). Together, these results suggest that (1) E-cadherin is less associated to neonatal cell-cell junctions in neonates than in adults and trafficked more in the cytosol; (2) $\beta$-catenin may be associated to other classical cadherins than E-cadherin in the neonatal intestinal epithelium, considering the brighter $\beta$-catenin signals than that of E-cadherin in neonatal junctions; and (3) the large pool of cytosolic $\beta$-catenin might reflect the activation of the Wnt pathway in the neonatal IB, relative to adults, as one would expect for a fast-growing tissue (Figures 3E, S4C, and S4D).

Wnt can activate the transcription of genes encoding E-cadherin repressors, such as Snai1 (Snail1), Snai2 (Slug), Zeb1 
(Zeb1), and Twist1 (Twist1), whose expression in mature epithelial tissues induces a depolarization process referred to as epithelial-to-mesenchymal transition (EMT). Activation of EMT factors is associated with a downregulation of E-cadherin expression and polarization and that of other junction proteins, as well as the upregulation of $\mathrm{N}$-cadherin gene (Cdh2) (Coopman and Djiane, 2016; Thiery et al., 2009). Indeed, transcript levels of Snai1, Snai2, Zeb1, Twist1, and Cdh2 are higher in the P5 small intestine (Figure 3F) and colon (Figure S4E) compared to that of adults, reflecting a higher Wnt activity in the neonatal intestinal epithelium and accounting for its incomplete epithelial polarization, which likely translates into the lower barrier function of the neonatal IB, as compared to adult mice.

\section{Age- and microbiota-dependent increased}

permissiveness of the neonatal GVB

Once the IB is crossed, GBS has to go through the GVB to access the systemic circulation and ultimately reach the central nervous system. We therefore investigated the respective impacts of age and microbiota on GVB permeability. We analyzed the expression of PLVAP (plasmalemma vesicle-associated protein), a structural component of endothelial fenestrae that regulates blood vessels' basal permeability, leukocyte migration, and angiogenesis; maintains the integrity of blood vessels; and is associated with increased GVB permissiveness (Guo et al., 2016; Spadoni et al., 2015; Wang et al., 2012). The ratio of PLVAP signal over endothelial marker CD31 signal showed that PLVAP was more intense in the P5 gut endothelium compared to adults, as well as in GF adult mice, compared to conventional adult mice (Figures 4A, 4B, S5A, and S5B). Quantification the Plvap gene transcript level in the intestine (Figure $4 \mathrm{C}$ ) and the colon (Figure S5D) confirmed these results. Claudin- 5 signal, a junctional protein of endothelial cells (Morita et al., 1999), was fainter in the $\mathrm{P} 5$ and GF adult villus endothelium than in conventional adult mice (Figures S5A and S5C). These results show that the GVB is more permissive in neonatal than in adult mice, and the agedependent maturation of the gut microbiota contributes to the enhancement of the barrier function of the GVB. This likely accounts for the overall higher translocation level of GBS into the systemic circulation in neonatal mice than in adult mice.

Microbiota and control of bacteremia and meningitis Following the GBS dissemination route from the gut to the brain, we next focused on the ability of neonates to control systemic infection. The gut microbiota is involved in maturation of systemic innate immune defenses (Deshmukh et al., 2014; Khosravi et al., 2014). We therefore compared the control of GBS bacteremia in neonatal and adult mice. To circumvent the expected bias due to differences in colonization resistance as well as IB and GVB permissiveness between neonatal and adult mice, we inoculated GBS intraperitoneally (i.p.). Bacterial burden in blood, liver, and brain decreased with age and was higher in GF adult than in conventional adult mice $24 \mathrm{~h}$ post-inoculation (Figure 4D), showing that the control of GBS systemic infection is age dependent and associated with mature microbiota. Consistent with these results, oral transfer of conventional adult microbiota played a protective role in GF adult (Figure 4E) and P15 mice (Figures S1C and 4F) toward GBS systemic and brain infection.
These results show that the mature microbiota is involved in control of GBS bacteremia, and their absence contributes to a higher systemic bacterial load and favor transfer of bloodborne GBS to the central nervous system in neonatal mice.

Age-dependent and microbiota-independent lower tightness of neonatal CPs

As a last potential host factor involved in modulating bacterial access to the brain, we finally investigated whether the neonatal BBB (BBB per se, $\mathrm{BMB}$, and $\mathrm{CP}$ ) is more permissive than that of adults. The lower ability of neonates to control GBS bacteremia may give rise to a higher GBS concentration upstream of the BBB and a subsequent increase in brain invasion. To avoid this potential bias, we performed $1 \mathrm{~h}$ i.p. infection, a short time frame where one expects minimal GBS multiplication and a negligible control of bacteremia by the immune system.

We observed GBS located (1) in the CP endothelium, upstream of the epithelial CP barrier; (2) within lateral ventricles; (3) in the BMB, within the subarachnoid space and in contact with the pia or with meningeal vessels; (4) in contact with vessels plunging from $\mathrm{BMB}$ into parenchyma; and (5) rarely within brain parenchyma (Figures 5A, 5B, and S5E). These observations suggest that GBS may cross the $\mathrm{CP}$ and reach the BMB via the CSF, where they accumulate, and cross the BMB endothelium. To assess this hypothesis, we developed a dynamic model of GBS crossing the three BBB interfaces (Figure $5 \mathrm{C}$ ). Based on GBS enumeration in all organs upon infection (Figures S5F and S5G), CP were predicted as being the main BBB interface targeted by GBS, implying that bacteria in the BMB came at least partially from the CP via the CSF. Furthermore, the flow of bacteria from blood to BBB interfaces decreased according to age (Figure 5D), suggesting that BBB permissiveness toward GBS is higher in neonates than in adults, in an age-dependent and microbiota-independent manner. Consistent with the model, $2 \mathrm{~h}$ after inoculation with Evans Blue, dye concentration was higher in the CSF of P5 mice, compared to adult mice (Figure 5E), suggesting that CP barrier function is weaker in neonates. Importantly, this effect is age dependent but microbiota independent (Figure 5E). Of note, $16 \mathrm{~h}$ post-i.p. inoculation, GBS burden decreased in liver and spleen, yet not significantly in blood, $\mathrm{BMB}$, and brain parenchyma of adult mice. It increased in P5 blood, liver, and parenchyma, yet not significantly in CP and BMB (Figure S6A). These results show that GBS infection is less controlled in P5 than in adult mice, in agreement with our previous results (Figure 4D).

Interestingly, we detected intracellular GBS-likely within immune cells - in contact with $\mathrm{CP}, \mathrm{BMB}$, and parenchyma vessels upstream and downstream of the endothelial barrier, as well as infection foci within perivascular spaces (Figure S6B). This suggests that after initial brain invasion by extracellular GBS through $\mathrm{CP}$ and $\mathrm{BMB}$, the ensuing immune response may favor a second wave of intracellular GBS invasion carried by recruited immune cells.

Low cohesiveness of neonatal CP junctions, Wnt pathway activity, and permissiveness to GBS

As for the intestinal epithelium, the higher permissiveness of neonatal $\mathrm{CP}$ may associate with a lower polarization of $\mathrm{CP}$ 
A
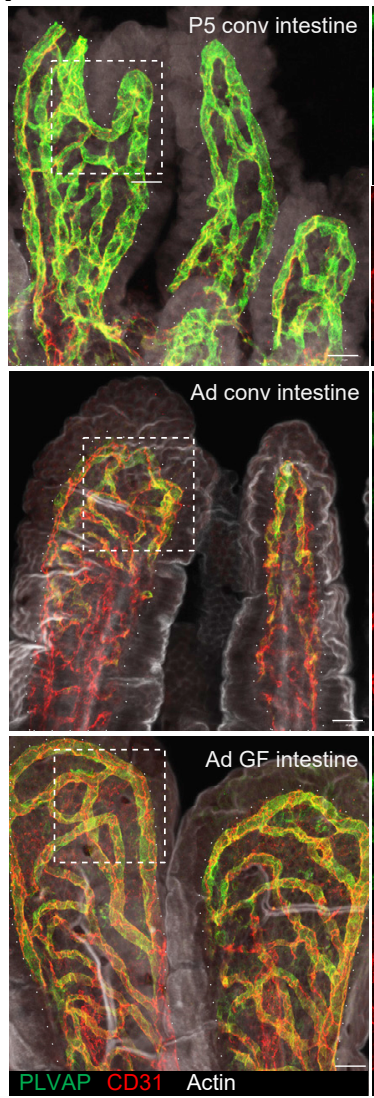

B
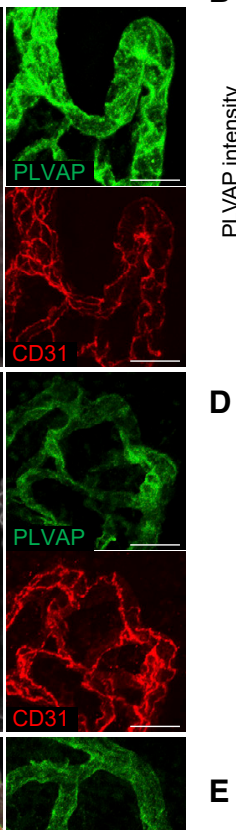

$\mathbf{E}$
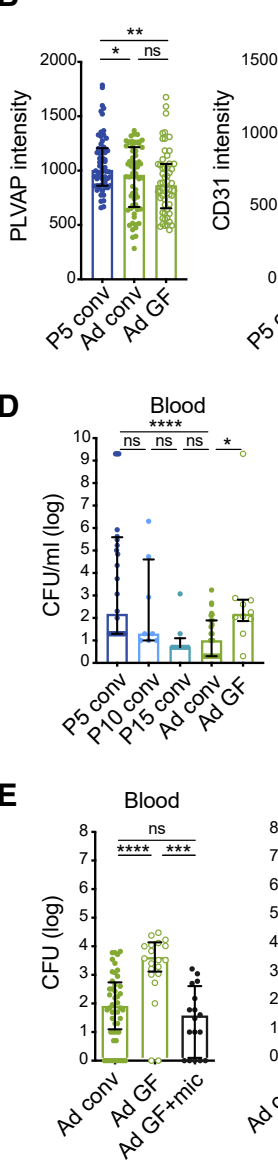
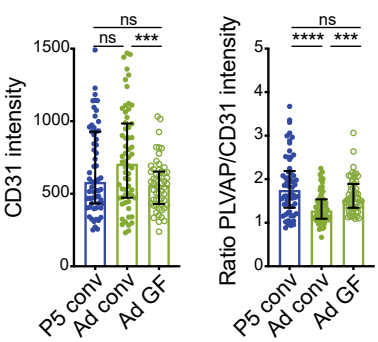

C
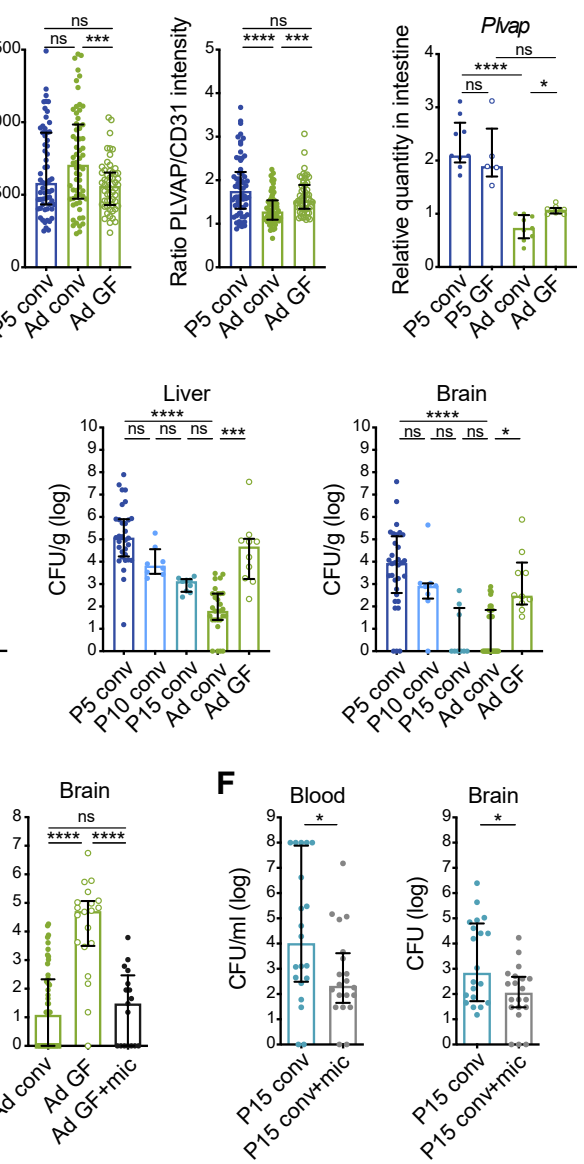

Figure 4. Higher permissiveness of the neonatal GVB and GBS systemic dissemination to the brain

(A) Confocal imaging and 9.64- $\mu$ m-thick maximum intensity projection of conventional and GF P5 and adult intestine sections stained for PLVAP and the endothelial marker CD31. Light gray dots delimit the endothelial area of each villus in which PLVAP and CD31 signals were quantified in (B). Scale bars, $10 \mu \mathrm{m}$. (B) PLVAP and CD31 intensity signals and ratio from (A) $(n=62$ for conventional P5 and adult, $n=56$ for adult GF).

(C) Relative expression of Plvap (Plvap) in the intestine of conventional P5 $(n=10)$ and adult $(n=9)$ and GF P5 ( $n=5)$ and adult $(n=10)$ mice.

(D) GBS count/ml of blood and per gram of organ of conventional P5 ( $n=32), P 10(n=8), P 15(n=9)$, and adult $(n=29)$ and of GF adult ( $n=10)$ mice 24 h post-i.p. inoculation with $2.5 \times 10^{5} \mathrm{GBS} / \mathrm{g}$ of mice.

(E) GBS count in blood and brain of conventional and GF adult mice $24 \mathrm{~h}$ post-intravenous inoculation with $5 \times 10^{6} \mathrm{GBS}$. Two weeks before inoculation, mice were orally inoculated with PBS (Ad conv, $n=48$; Ad GF, $n=19$ ) or with intestinal luminal content of conventional adult mice (Ad GF+mic, $n=17$ ).

(F) GBS count in blood and brain of conventional P15 mice $24 \mathrm{~h}$ post-i.p. inoculation with $10^{8} \mathrm{GBS}$. Before oral inoculation, mice were inoculated at P3, P6, and P9 with PBS (P15 conv) or with intestinal luminal content of conventional adult mice (P15 conv+mic) ( $n=20)(S e e$ Figure S1B).

Statistical tests: Brown-Forsythe and Welch ANOVA (B and C), Kruskal-Wallis (D and E), Mann-Whitney (F). Median \pm IQR.

epithelial junctions. Adult CP cells have a larger transversal section than do neonates (Figures 6A, S7A, and S7B), and as a consequence, there are more epithelial junctions per surface unit in P5 CP than in that of adults (Figure S7C), a factor that could increase the odds of paracellular bacterial translocation through neonatal CP junctions. Adult CP epithelial junctions also look more structured and convoluted, as they exhibit a higher length per cell surface than do neonates (Figures 6A$6 \mathrm{C})$. In contrast, P5 CP epithelial cells look rounder with shorter junctions per cell surface, which also appear to be locally disjoined (Figures 6A-6D, arrows). E-cadherin labeling is also more apical and intermixed with Zonula Occludens-1 (ZO-1) signal in neonates, whereas in adult mice it is - as expected for mature junctions-below ZO-1 and colocalizes with that of $\beta$-catenin (Figure $6 \mathrm{C}$ ). These observations suggest that AJs and TJs are not mature in neonatal CP. Consistent with these results, ZO-1 repartition is also irregular along P5 junctions, compared to adults (Figures 6B and S7D), and E-cadherin, claudin-1, and occludin are also significantly less expressed in P5 CP than in adults (Figures S7E and S7F). Altogether, these results suggest that neonatal $\mathrm{CP}$ epithelial cell junctions are not as polarized and cohesive as in adults, and this may reflect a higher Wnt activity, as expected for a fast-growing tissue (Figures 6E and S7G). Indeed, the amount of $\beta$-catenin protein (Figure S7F), transcript levels of cell-cycle factors Ccnd1 (cyclin-D1) and Ccnd2 (cyclin-D2) (Figure $\mathrm{S} 7 \mathrm{H}$ ) that are activated by the Wnt 
A
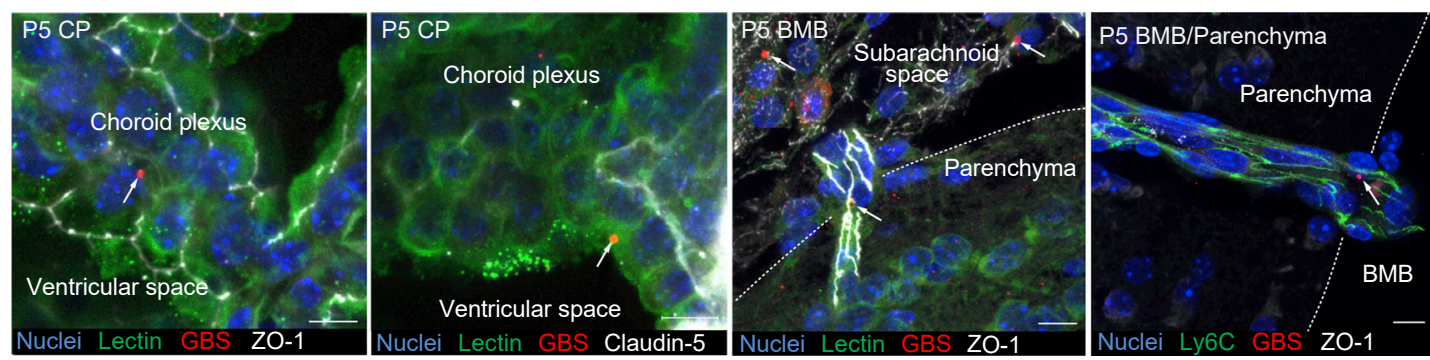

B
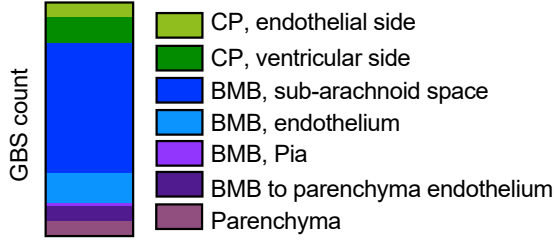

Total $=63$
D

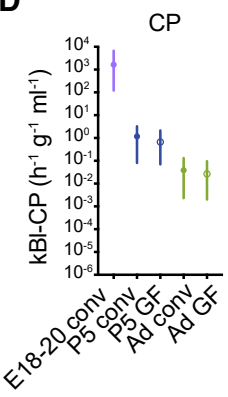

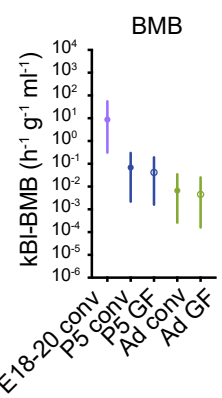

BMB

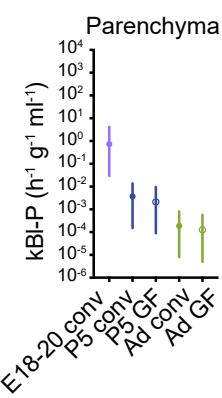

E

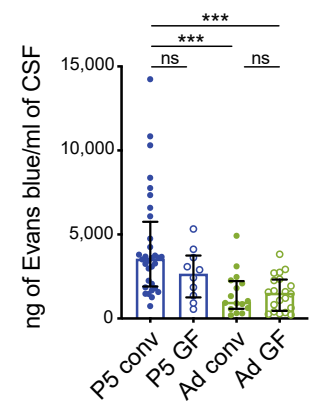

Figure 5. Higher permissiveness of the neonatal CP barrier

(A) Confocal imaging and 8- $\mu$ m-thick maximal intensity projections of conventional P5 brain sections $1 \mathrm{~h}$ post-i.p. inoculation with $10^{6} \mathrm{GBS} / \mathrm{g}$ of mice. Sections were stained for nuclei; surface of CP; endothelial and parenchyma cells with Lycopersicon esculentum lectin (Lectin); or myeloid cells/endothelium Ly6C, GBS, and ZO-1 or claudin-5 (CLD5). White arrows, GBS. Scale bars, $10 \mu \mathrm{m}$.

(B) Repartition of GBS detected by microscopy in P5 brain $1 \mathrm{~h}$ post-i.p. inoculation with $10^{6} \mathrm{GBS} / \mathrm{g}$ of mice.

(C) Mathematical model for clearance and flow by efflux and influx of GBS between mice organs $1 \mathrm{~h}$ post-i.p. inoculation with $10^{6}$ GBS/g of mice. The overall clearance occurring between the inoculation and the sacrifice is represented by the death parameter. BMB is connected to CP as well as parenchyma to BMB by the CSF. The connections among the organs and the associated rate constants $(\mathrm{k})$ are diagrammed.

(D) Rate constants of the GBS movements from blood to CP (kBI-CP), BMB (kBI-BMB), and parenchyma (kBI-P) in conventional E18-E20 $(n=21)$, P5 ( $n=48)$, and adult $(n=33)$ and in GF P5 $(n=31)$ and adult $(n=28)$ mice 1 h post-i.p. inoculation with $10^{6} \mathrm{GBS} / \mathrm{g}$ of mice.

(E) Evans blue concentration in CSF of conventional P5 $(n=32)$ and adult $(n=16)$ and GF P5 $(n=10)$ and adult $(n=20)$ mice 2 h post-i.p. inoculation with 0.2 mg/g of mice.

Statistical test: Brown-Forsythe and Welch ANOVA (E). Median \pm IQR.

pathway (Thiery et al., 2009), and EMT factors genes Snai1, Snai2, Zeb1, Twist1, and Cdh2 (Figure 6F) were higher in P5 $\mathrm{CP}$, compared to adults.

Kim et al. (2015) have shown that GBS infection increases Snail1 expression and lowers TJ protein expression in endothelial cultured cells and zebrafish, associated with higher BBB permissiveness. Quantification of the expression of EMT factors genes Snai1 and Snai2 and TJ and AJ proteins genes in CP, $24 \mathrm{~h}$ post-oral inoculation with GBS, showed a higher expression of Ocln and a lower expression of Snai2 and Cldn5 (claudin-5) (Fig- ure S8A). As claudin-5 is involved specifically in endothelial TJs (Steinemann et al., 2016), these results suggest that GBS infection does not significantly modify CP epithelium permissiveness but may increase CP endothelium leakiness, which could favor the translocation in the brain parenchyma of GBS-infected immune cells and amplify brain infection.

To confirm that the lower polarization of the neonatal CP can drive permissiveness to GBS, we treated neonatal mice with metformin, which has been shown to favor the assembly and the stabilization of junction proteins at epithelial cell-cell 
A

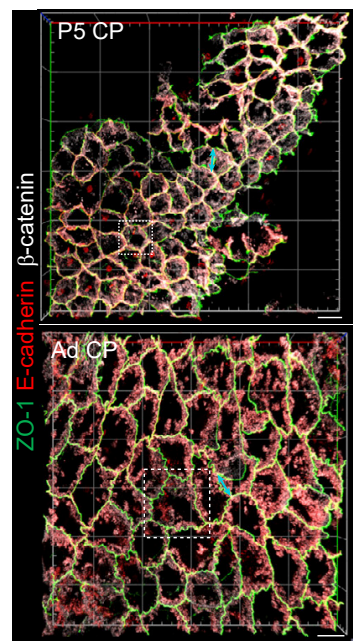

B

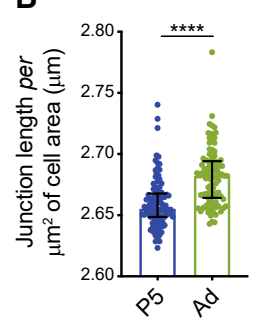

C

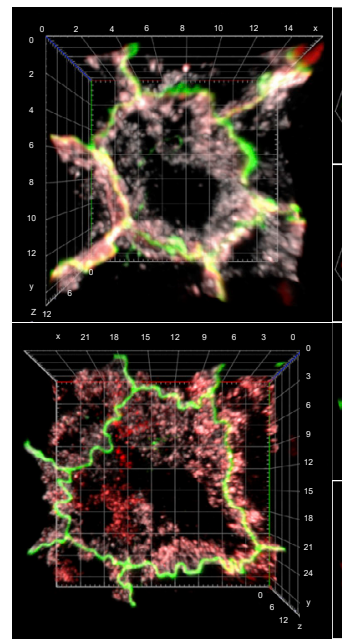

D

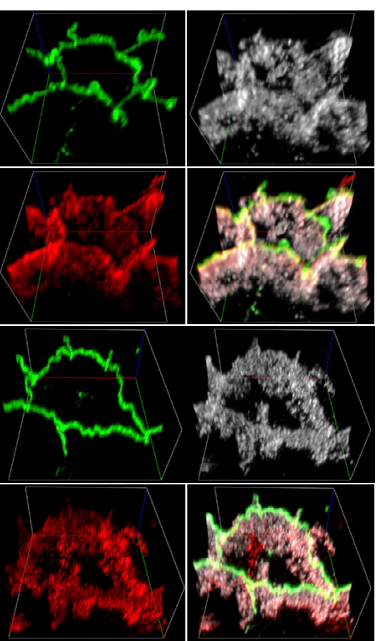

D

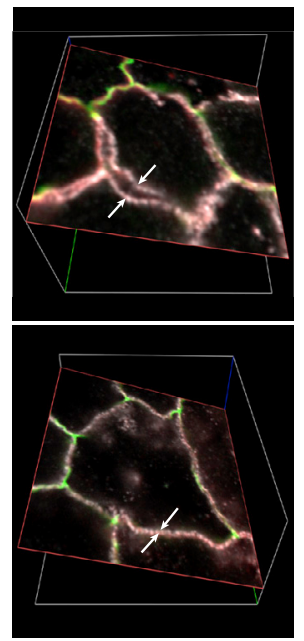

E

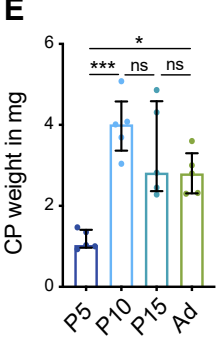

$\mathbf{F}$

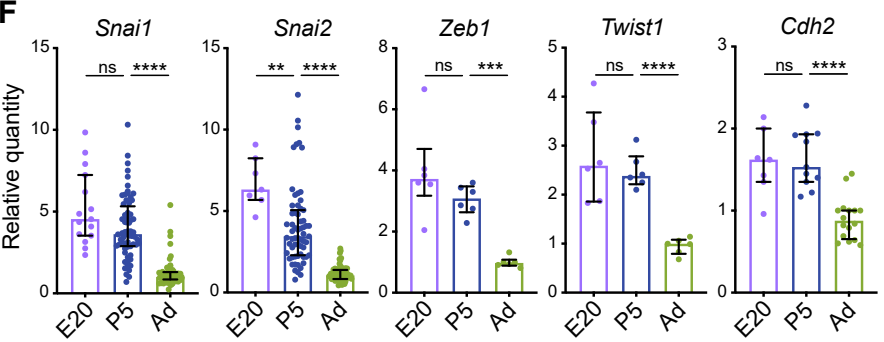

Figure 6. Higher Wnt activity in neonatal CP is associated with lower polarization of epithelial junctions

(A) Confocal imaging and 9- $\mu \mathrm{m}$-thick maximal intensity 3D projections of whole CP from conventional P5 and adult mice. CP were stained for $\beta$-catenin, Ecadherin, and ZO-1. Light blue double arrows indicate junctions along which ZO-1 intensity repartition was analyzed in Figure S5D. Scale bars, 10 $\mu \mathrm{m}$.

(B) Ratio of the perimeter over the cell surface of 100 P5 versus adult CP cells.

(C) 3D reconstruction view of $\mathrm{PC}$ cell from inset in (A): top angle 0 view (left panel) and around angle $25^{\circ}$ tilted view (right panels).

(D) Planal clipping view (yz view) of CP cells in (C). Arrows point at membranes that appeared interspaced in P5.

(E) CP weight in P5, P10, P15, and adult mice $(n=5)$.

(F) Relative expression of Snai1 (Snail1), Snai2 (Slug), Zeb1 (Zeb1), Twist1 (Twist1), and Cdh2 (N-cadherin) in the CP of conventional E18-E20 ( $\mathrm{n}=15)$, P5 ( $\mathrm{n}=64$ ), and adult $(\mathrm{n}=52)$ mice.

Statistical tests: Mann-Whitney (B), one-way ANOVA (E), Brown-Forsythe and Welch ANOVA (F). Median \pm IQR.

junctions (Banerjee et al., 2016; Kalsi et al., 2019). After $48 \mathrm{~h}$ of metformin oral daily inoculation, transcript levels of genes coding for cell junction proteins in gut tissues were not affected, with the exception of Ocln expression, which was increased in the colon (Figures S8B and S8C). However, we observed that P5 CP junctions were more structured in metformin-treated than in control P5 mice (Figure 7A). This correlates with an increase of Cdh1, Cldn3, Cldn1 (claudin-1), Ocln, and Tjp1 (ZO-1) transcript level in metformin-treated CP (Figure 7B), suggesting that metformin may increase the barrier function of neonatal CP. Sixteen hours after oral inoculation with GBS, metformin-treated neonates harbored equivalent bacterial burden in intestine, colon, blood, liver, and spleen but decreased bacterial burden in $\mathrm{CP}$ as compared to mock treated (Figures $7 \mathrm{C}$ and S8D), consistent with a stabilizing effect of metformin on CP junctions leading to reducing permissiveness of $\mathrm{CP}$ toward GBS. Of note, we also observed a trend for a reduced GBS burden in BMB and parenchyma after metformin-treatment. This suggests that decreased permissiveness of the CP to
GBS reduces GBS number in BMB and brain parenchyma, in agreement with our dynamic model of GBS crossing of the BBB interfaces, but to a lesser extent than in CP.

Altogether, these results reveal that the lower expression and polarization of CP junction proteins, consecutive to Wnt activity in the context of the continuing growth and maturation of $\mathrm{CP}$ in neonatal mice, correlate with increased permissiveness of neonatal CP and susceptibility to GBS meningitis (see recapitulative model in Figure S8E).

\section{DISCUSSION}

Here, we investigated the reasons of neonatal susceptibility to bacterial meningitis and identified the respective roles of microbiota maturation and age per se in its development. We have shown that in neonates, the absence of mature microbiota accounts in part for their higher susceptibility to GBS meningitis. It eases colonization of the gut lumen by GBS and its translocation across the GVB and favors bacteremia. We have also 

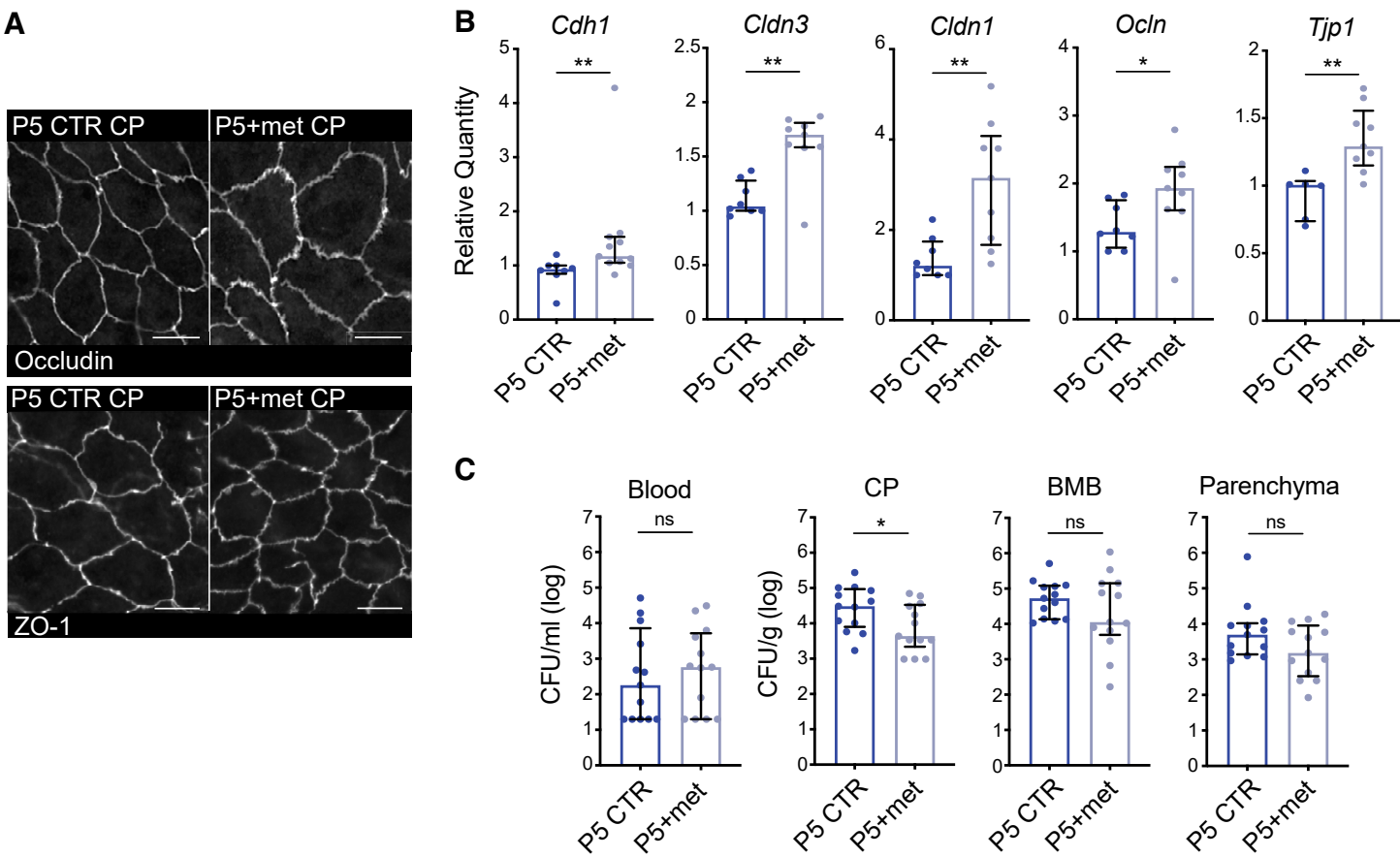

Figure 7. Increased expression of genes coding for CP epithelial AJ and TJ proteins is associated with reduced crossing of CP by GBS (A) Confocal imaging and 0.8- $\mu$ m-thick maximal intensity projections of brain sections from conventional P5 (P5 CTR) and metformin-treated conventional P5 (P5+met) mice. Sections were stained for occludin or ZO-1. Scale bars, $10 \mu \mathrm{m}$.

(B) Relative expression of Cdh1 (E-cadherin), Cldn3 (claudin-3), Cldn1 (claudin-1), Ocln (occludin), and Tjp1 (ZO-1) in the CP of conventional P5 (P5 CTR, n = 8 ) and metformin-treated conventional P5 (P5+met, $\mathrm{n}=11)$ mice.

(C) GBS count in blood, CP, BMB, and parenchyma of conventional P5 (P5 CTR, $\mathrm{n}=18$ ) and metformin-treated conventional P5 (P5+met, $\mathrm{n}=17) 16 \mathrm{~h}$ post-oral inoculation with $10^{7} \mathrm{GBS} / \mathrm{g}$ of mice.

Statistical test: Mann-Whitney (B and C). Median \pm IQR.

demonstrated that both the epithelial IB and CP are more permissive in neonates than in adults, in an age-dependent but microbiota-independent manner. The polarization of neonatal IB and CP epithelial junctions is weaker than in adults, owing to higher Wnt pathway activity, which favors GBS translocation across the IB and CP.

Neonatal microbiota immaturity promotes gut lumen colonization by GBS. Indeed, the absence of mature microbiota eases colonization of the lumen by GBS, which can multiply with fewer competitors than in the presence of diverse and balanced mature microbiota, with more readily available nutrients and lower antibacterial defenses secreted by the intestinal tissue (Buffie and Pamer, 2013; Keith and Pamer, 2019). Weak resistance to colonization thus allows for a higher GBS inoculum in the gut lumen, leading to its subsequent proportional increased crossing of the IB and systemic dissemination. Neonatal microbiota immaturity is therefore a major susceptibility factor for LOD. In line with this finding, the higher incidence of CC17 GBS in human LOD compared to other GBS is associated with its greater intestinal colonization ability (Tazi et al., 2019, 2010). Conventionalization of neonatal mice with mature microbiota protects mice from GBS infection after both oral and i.p. inoculation. This shows that in addition to the colonization resistance toward GBS, mature microbiota is crucial for bacteremia control, a critical factor for central nervous system bacterial invasion.
Whereas protection of neonatal mice toward GBS infection through gut colonization resistance and maturation of the immune system may be triggered specifically by 1 or some of the 11 commensals OTUs out of the 12 that are enriched in the gut lumen content of conventionalized neonates, microbiota diversity itself is likely the key parameter (Buffie and Pamer, 2013; Keith and Pamer, 2019). The gut microbiota plays a critical role in the maturation of multiple features of the immune system in mice, but also in humans (Deshmukh et al., 2014; Gensollen et al., 2016; Khosravi et al., 2014). Accordingly, prolonged neonatal administration of antibiotics increases the risk of sepsis in mice and humans (Kuppala et al., 2011). Furthermore, the gut microbiota has been shown to enhance myelopoiesis (Khosravi et al., 2014), and in neonates, the installing microbiota triggers granulocytosis and contribute to better control of $E$. coli $\mathrm{K} 1$ sepsis (Deshmukh et al., 2014). However, immature neutrophils circulate in neonates, rendering the overall phagocytic performance of neonatal neutrophils weaker than in adults (Makoni et al., 2016), which may also contribute to the lower ability of neonates to control bacteremia. Lower colonization resistance and immune system immaturity due to neonatal gut microbiota composition, therefore, accounts at least partly for GBS susceptibility in neonates, both in humans and in mice.

The role of commensal bacteria in the maturation of gut tissues has been previously established. Indeed, the intestinal microbiota 
promotes (1) the resistance to gut lumen colonization (Buffie and Pamer, 2013; Keith and Pamer, 2019); (2) the synthesis, structure, and penetrability of the protective mucus layer (Johansson et al., 2015); (3) the synthesis of microbe-specific immunoglobulins; and (4) the maturation of the mucosal immune system (Belkaid and Hand, 2014; Fulde and Hornef, 2014; Jain and Walker, 2015; Chin et al., 2017). In addition, we identified that gut microbiota influences the permissiveness of the GVB, consistent with a previous study that showed that the gut microbiota regulate intestinal angiogenesis (Stappenbeck et al., 2002). Indeed, PLVAP expression increases upon induction of angiogenesis that is stimulated by vascular-endothelial growth factor (Guo et al., 2016). However, in agreement with Thevaranjan et al. (2017), using young and healthy adult mice, we detected a microbiota-independent role in IB permeability to FITC-Dextran. Indeed, we showed a 5-fold increase of FITC-Dextran diffusion through IB in neonatal GF compared to adult GF mice, which is age dependent and microbiota independent. We cannot exclude that mature microbiota would lead to a decrease of permissiveness in P5 neonates, although it is not experimentally testable, given the time needed for mature microbiota to establish. While the rat colon transepithelial permeability has been reported to be higher in neonates than in adults (Mullin et al., 2002), our study demonstrates that the neonatal IB is less polarized and cohesive than in adults and that this relates to tissue growth and development.

We evidenced that both neonatal intestinal and CP epithelia are characterized by a higher Wnt activity than in adults, which is consistent with the lower degree of polarization and maturation of cell-cell junctions in the context of tissues' and organs' growth and plasticity (Coopman and Djiane, 2016; Thiery et al., 2009). This higher Wnt activity of the neonatal IB and CP epithelia is in line with the growing and developing nature of organs, whose size increases dramatically within a couple of weeks. CP weight increases 4-fold between P5 and P10 and is stable afterward, suggesting that Wnt activity peaks between P5 and $\mathrm{P} 10$, at a time when CSF production and nutrient transport through CP is needed for brain development (Saunders et al., 2018). The observation that the polarization, structure, and cohesiveness of the neonatal IB and CP epithelial junctions are weaker than in adults is consistent with the profound phenotypic changes associated with Wnt activation, which include the loss of cell-cell adhesion, the loss of cell polarity, and the acquisition of migratory and invasive properties (Coopman and Djiane, 2016; Thiery et al., 2009). The age-dependent growth and development of the intestinal and CP epithelia is thus likely responsible for their relatively weaker barrier properties, which favors GBS translocation across host epithelial barriers and ultimately neonatal susceptibility to meningitis. GBS infection has been shown to trigger the activation of EMT in endothelial cells, impede the expression of TJ proteins, and promote endothelial permeability, allowing GBS dissemination in the adult zebrafish (Kim et al., 2015). Therefore, in neonates, in agreement with our observation that GBS infection itself reduces claudin-5 expression in CP, GBS may also decrease GVB and/or brain microvessel barrier functions - thereby further amplifying neonatal susceptibility to GBS meningitis - and favor the crossing of recruited infected immune cells into brain perivascular spaces. In humans, although neonatal TJs are more mature at birth than in mice, they also undergo a major maturation process (Jain and Walker, 2015; Chin et al., 2017; Saunders et al., 2018; Liddelow, 2015); therefore higher Wnt activity in neonates may also account for increased neonatal susceptibility to GBS in humans.

Neonates are generally considered more susceptible to infection than adults because of the immaturity of their immune systems. Here, we have shown that intestinal microbiota immaturity and epithelial barriers' permissiveness promote bacterial dissemination from the gut to the brain and collectively account for neonatal susceptibility to bacterial meningitis. While modulating neonatal developmental pathways to reduce the intrinsic permissiveness of neonatal epithelial barriers would likely induce multiple detrimental effects, maturing the neonatal microbiota with the administration of probiotics and/or prebiotics may help prevent neonatal bacterial meningitis by promoting the maturation of neonatal intestinal defense.

\section{STAR $\star$ METHODS}

Detailed methods are provided in the online version of this paper and include the following:

- KEY RESOURCES TABLE

- RESOURCE AVAILABILITY

○ Lead contact

○ Materials availability

○ Data and code availability

- EXPERIMENTAL MODEL AND SUBJECT DETAILS

○ Animals

O Bacteria

- METHOD DETAILS

O Mice treatments and infections

O Intestinal and CP permeability assays

O Immunofluorescence

Mathematical modeling

O Quantitative real-time PCR (qRT-PCR)

Western blotting

Microbiota analysis

- QUANTIFICATION AND STATISTICAL ANALYSIS

Image analysis

Data and statistical analysis

\section{SUPPLEMENTAL INFORMATION}

Supplemental information can be found online at https://doi.org/10.1016/j. celrep.2021.109319.

\section{ACKNOWLEDGMENTS}

We thank Dmitry Ershov from the Bioinformatics and Biostatistics Hub (Institut Pasteur, USR 3756 CNRS) and the Image Analysis Hub for their help with the image and/or data analysis. We thank George M. Haustant and Thomas Cokelaer (Biomics Platform, C2RT, Institut Pasteur, Paris, France, supported by France Génomique [ANR-10-INBS-09-09] and IBiSA) for 16S sequencing. We thank Claire Poyart for the BM110 GBS strain and Ivan Gladwyn-Ng and Laurent Nguyen for helpful discussions and preliminary experiments. We thank the members of the Biology of Infection Unit for their support, Sylvain Levallois for critical reading, Rémy Dailleux and Thifaine Pouillon for help, and the Institut Pasteur Animalerie Centrale and Gnotobiology Center. This work was supported financially by Institut Pasteur, the Major Federating Program Microbes 
and Brain of Institut Pasteur, Labex IBEID, and Institut National de la Santé et de la Recherche Médicale. L.H. is supported by the Pasteur-Paris University (PPU) International PhD Program, funded by the European Union's Horizon 2020 research and innovation program under the Marie Sklodowska-Curie grant agreement 665807 and the "Ecole Doctorale FIRE-Programme Bettencourt" of the CRI Paris. M.L. ORCID: 0000-0002-4491-1063. M.L. is a senior member of Institut Universitaire de France.

\section{AUTHOR CONTRIBUTIONS}

M.L. conceptualized and supervised the project, with input from L.T.; L.T., M.A., L.H., and O.D. performed the experiments and A.A. the modeling study. L.T., M.A., A.A., L.H., S.C., and M.L. analyzed the data. P.-M.L. and S.C. provided mentorship. L.T. wrote the first draft, which was edited by M.L. All co-authors commented on it.

\section{DECLARATION OF INTERESTS}

The authors declare no competing interests.

Received: September 21, 2020

Revised: March 25, 2021

Accepted: June 7, 2021

Published: June 29, 2021

\section{REFERENCES}

Al Nabhani, Z., Dulauroy, S., Marques, R., Cousu, C., Al Bounny, S., Déjardin, F., Sparwasser, T., Bérard, M., Cerf-Bensussan, N., and Eberl, G. (2019). A Weaning Reaction to Microbiota Is Required for Resistance to Immunopathologies in the Adult. Immunity 50, 1276-1288.e5.

Albanese, D., Fontana, P., De Filippo, C., Cavalieri, D., and Donati, C. (2015). MICCA: a complete and accurate software for taxonomic profiling of metagenomic data. Sci. Rep. 5, 9743.

Badri, M.S., Zawaneh, S., Cruz, A.C., Mantilla, G., Baer, H., Spellacy, W.N., and Ayoub, E.M. (1977). Rectal colonization with group B streptococcus: relation to vaginal colonization of pregnant women. J. Infect. Dis. 135, 308-312.

Banerjee, P., Dutta, S., and Pal, R. (2016). Dysregulation of Wnt-Signaling and a Candidate Set of miRNAs Underlie the Effect of Metformin on Neural Crest Cell Development. Stem Cells 34, 334-345.

Belkaid, Y., and Hand, T.W. (2014). Role of the microbiota in immunity and inflammation. Cell 157, 121-141.

Bhatt, T., Rizvi, A., Batta, S.P.R., Kataria, S., and Jamora, C. (2013). Signaling and mechanical roles of E-cadherin. Cell Commun. Adhes. 20, 189-199.

Bingen, E., Denamur, E., Lambert-Zechovsky, N., Aujard, Y., Brahimi, N., Geslin, P., and Elion, J. (1992). Analysis of DNA restriction fragment length polymorphism extends the evidence for breast milk transmission in Streptococcus agalactiae late-onset neonatal infection. J. Infect. Dis. 165, 569-573.

Braniste, V., Al-Asmakh, M., Kowal, C., Anuar, F., Abbaspour, A., Tóth, M., Korecka, A., Bakocevic, N., Ng, L.G., Kundu, P., et al. (2014). The gut microbiota influences blood-brain barrier permeability in mice. Sci. Transl. Med. 6, $263 r a 158$.

Bray, J.R., and Curtis, J.T. (1957). An Ordination of the Upland Forest Communities of Southern Wisconsin. Ecol. Monogr. 27, 325-349.

Buffie, C.G., and Pamer, E.G. (2013). Microbiota-mediated colonization resistance against intestinal pathogens. Nat. Rev. Immunol. 13, 790-801.

Chin, A.M., Hill, D.R., Aurora, M., and Spence, J.R. (2017). Morphogenesis and maturation of the embryonic and postnatal intestine. Semin. Cell Dev. Biol. 66, 81-93.

Coopman, P., and Djiane, A. (2016). Adherens Junction and E-Cadherin complex regulation by epithelial polarity. Cell. Mol. Life Sci. 73, 3535-3553.

Deshmukh, H.S., Liu, Y., Menkiti, O.R., Mei, J., Dai, N., O’Leary, C.E., Oliver, P.M., Kolls, J.K., Weiser, J.N., and Worthen, G.S. (2014). The microbiota reg- ulates neutrophil homeostasis and host resistance to Escherichia coli $\mathrm{K}$ sepsis in neonatal mice. Nat. Med. 20, 524-530.

Disson, O., and Lecuit, M. (2012). Targeting of the central nervous system by Listeria monocytogenes. Virulence 3, 213-221.

Disson, O., Nikitas, G., Grayo, S., Dussurget, O., Cossart, P., and Lecuit, M (2009). Modeling human listeriosis in natural and genetically engineered animals. Nat. Protoc. 4, 799-810.

Edgar, R.C., and Flyvbjerg, H. (2015). Error filtering, pair assembly and error correction for next-generation sequencing reads. Bioinformatics 31, 34763482. https://doi.org/10.1093/BIOINFORMATICS/BTV401.

Edmond, K.M., Kortsalioudaki, C., Scott, S., Schrag, S.J., Zaidi, A.K., Cousens, S., and Heath, P.T. (2012). Group B streptococcal disease in infants aged younger than 3 months: systematic review and meta-analysis. Lancet $379,547-556$.

Fulde, M., and Hornef, M.W. (2014). Maturation of the enteric mucosal innate immune system during the postnatal period. Immunol. Rev. 260, 21-34.

Gensollen, T., Iyer, S.S., Kasper, D.L., and Blumberg, R.S. (2016). How colonization by microbiota in early life shapes the immune system. Science 352, 539-544.

Gilks, W.R., Richardson, S., and Spiegelhalter, D. (1996). Markov Chain Monte Carlo in Practice (Chapman \& Hall).

Guo, L., Zhang, H., Hou, Y., Wei, T., and Liu, J. (2016). Plasmalemma vesicleassociated protein: A crucial component of vascular homeostasis. Exp. Ther. Med. 12, 1639-1644

Hansen, S.M., Uldbjerg, N., Kilian, M., and Sørensen, U.B.S. (2004). Dynamics of Streptococcus agalactiae colonization in women during and after pregnancy and in their infants. J. Clin. Microbiol. 42, 83-89.

Hill, M.O. (1973). Diversity and Evenness: A Unifying Notation and Its Consequences. Ecology 54, 427-432.

Hooper, L.V., Wong, M.H., Thelin, A., Hansson, L., Falk, P.G., and Gordon, J.I. (2001). Molecular analysis of commensal host-microbial relationships in the intestine. Science 291, 881-884.

Jain, N., and Walker, W.A. (2015). Diet and host-microbial crosstalk in postnatal intestinal immune homeostasis. Nat. Rev. Gastroenterol. Hepatol. 12, 14-25.

Johansson, M.E.V., Jakobsson, H.E., Holmén-Larsson, J., Schütte, A., Ermund, A., Rodríguez-Piñeiro, A.M., Arike, L., Wising, C., Svensson, F., Bäckhed, F., and Hansson, G.C. (2015). Normalization of host intestinal mucus layers requires long-term microbial colonization. Cell Host Microbe 18 , 582-592.

Kalsi, K.K., Garnett, J.P., Patkee, W., Weekes, A., Dockrell, M.E., Baker, E.H., and Baines, D.L. (2019). Metformin attenuates the effect of Staphylococcus aureus on airway tight junctions by increasing PKC -mediated phosphorylation of occludin. J. Cell. Mol. Med. 23, 317-327.

Keith, J.W., and Pamer, E.G. (2019). Enlisting commensal microbes to resist antibiotic-resistant pathogens. J. Exp. Med. 216, 10-19.

Khosravi, A., Yáñez, A., Price, J.G., Chow, A., Merad, M., Goodridge, H.S., and Mazmanian, S.K. (2014). Gut microbiota promote hematopoiesis to control bacterial infection. Cell Host Microbe 15, 374-381.

Kim, K.S. (2010). Acute bacterial meningitis in infants and children. Lancet Infect. Dis. 10, 32-42.

Kim, B.J., Hancock, B.M., Bermudez, A., Del Cid, N., Reyes, E., van Sorge, N.M., Lauth, X., Smurthwaite, C.A., Hilton, B.J., Stotland, A., et al. (2015). Bacterial induction of Snail1 contributes to blood-brain barrier disruption. J. Clin. Invest. 125, 2473-2483.

Kuppala, V.S., Meinzen-Derr, J., Morrow, A.L., and Schibler, K.R. (2011). Prolonged initial empirical antibiotic treatment is associated with adverse outcomes in premature infants. J. Pediatr. 159, 720-725.

Lemichez, E., Lecuit, M., Nassif, X., and Bourdoulous, S. (2010). Breaking the wall: targeting of the endothelium by pathogenic bacteria. Nat. Rev. Microbiol. 8, 93-104. 
Liddelow, S.A. (2015). Development of the choroid plexus and blood-CSF barrier. Frontiers in Neuroscience 9, 1-13. https://doi.org/10.3389/fnins.2015. 00032.

Love, M.I., Huber, W., and Anders, S. (2014). Moderated estimation of fold change and dispersion for RNA-seq data with DESeq2. Genome Biol. 15, 550.

Makoni, M., Eckert, J., Anne Pereira, H., Nizet, V., and Lawrence, S.M. (2016). Alterations in neonatal neutrophil function attributable to increased immature forms. Early Hum. Dev. 103, 1-7.

McMurdie, P.J., and Holmes, S. (2015). Shiny-phyloseq: Web application for interactive microbiome analysis with provenance tracking. Bioinformatics $31,282-283$

Morita, K., Sasaki, H., Furuse, M., and Tsukita, S. (1999). Endothelial claudin: claudin-5/TMVCF constitutes tight junction strands in endothelial cells. J. Cell Biol. 147, 185-194.

Mullin, J.M., Valenzano, M.C., Verrecchio, J.J., and Kothari, R. (2002). Ageand diet-related increase in transepithelial colon permeability of Fischer 344 rats. Dig. Dis. Sci. 47, 2262-2270.

Oksanen, A.J., Blanchet, F.G., Kindt, R., Legendre, P., Minchin, P.R., O'Hara, R.B., Simpson, G.L., Solymos, P., Stevens, M.H.H., et al. (2012). vegan: Community Ecology Package (R Project).

Press, W.H., Flannery, B.P., Teukolsky, S.A., and Vetterling, W.T. (1992). Numerical Recipes in C (Cambridge University Press).

Rognes, T., Flouri, T., Nichols, B., Quince, C., and Mahé, F. (2016). VSEARCH: a versatile open source tool for metagenomics. PeerJ 4, e2584.

Romain, A.S., Cohen, R., Plainvert, C., Joubrel, C., Béchet, S., Perret, A., Tazi, A., Poyart, C., and Levy, C. (2018). Clinical and Laboratory Features of Group B Streptococcus Meningitis in Infants and Newborns: Study of 848 Cases in France, 2001-2014. Clin. Infect. Dis. 66, 857-864.

Salazar, N., Arboleya, S., Valdés, L., Stanton, C., Ross, P., Ruiz, L., Gueimonde, M., and de Los Reyes-Gavilán, C.G. (2014). The human intestinal microbiome at extreme ages of life. Dietary intervention as a way to counteract alterations. Front. Genet. 5, 406.

Saunders, N.R., Dziegielewska, K.M., Møllgård, K., and Habgood, M.D. (2018). Physiology and molecular biology of barrier mechanisms in the fetal and neonatal brain. J. Physiol. 596, 5723-5756.
Sonnenburg, J.L., and Bäckhed, F. (2016). Diet-microbiota interactions as moderators of human metabolism. Nature 535, 56-64.

Soriani, M., Santi, I., Taddei, A., Rappuoli, R., Grandi, G., and Telford, J.L. (2006). Group B Streptococcus crosses human epithelial cells by a paracellular route. J. Infect. Dis. 193, 241-250.

Spadoni, I., Zagato, E., Bertocchi, A., Paolinelli, R., Hot, E., Di Sabatino, A., Caprioli, F., Bottiglieri, L., Oldani, A., Viale, G., Penna, G., et al. (2015). A gut-vascular barrier controls the systemic dissemination of bacteria. Science 350, 830-834.

Stappenbeck, T.S., Hooper, L.V., and Gordon, J.I. (2002). Developmental regulation of intestinal angiogenesis by indigenous microbes via Paneth cells. Proc. Natl. Acad. Sci. USA 99, 15451-15455.

Steinemann, A., Galm, I., Chip, S., Nitsch, C., and Maly, I.P. (2016). Claudin-1, -2 and -3 are selectively expressed in the epithelia of the choroid plexus of the mouse from early development and into adulthood while claudin- 5 is restricted to endothelial cells. Front. Neuroanat. 10, 16.

Tazi, A., Disson, O., Bellais, S., Bouaboud, A., Dmytruk, N., Dramsi, S., Mistou, M.Y., Khun, H., Mechler, C., Tardieux, I., et al. (2010). The surface protein HvgA mediates group B streptococcus hypervirulence and meningeal tropism in neonates. J. Exp. Med. 207, 2313-2322.

Tazi, A., Plainvert, C., Anselem, O., Ballon, M., Marcou, V., Seco, A., El Alaoui, F., Joubrel, C., El Helali, N., Falloukh, E., et al. (2019). Risk Factors for Infant Colonization by Hypervirulent CC17 Group B Streptococcus: Toward the Understanding of Late-onset Disease. Clin. Infect. Dis. 69, 1740-1748.

Thevaranjan, N., Puchta, A., Schulz, C., Naidoo, A., Szamosi, J.C., Verschoor, C.P., Loukov, D., Schenck, L.P., Jury, J., Foley, K.P., et al. (2017). Age-Associated Microbial Dysbiosis Promotes Intestinal Permeability, Systemic Inflammation, and Macrophage Dysfunction. Cell Host Microbe 21, 455-466.e4.

Thiery, J.P., Acloque, H., Huang, R.Y.J., and Nieto, M.A. (2009). Epithelialmesenchymal transitions in development and disease. Cell 139, 871-890.

Wang, Q., Garrity, G.M., Tiedje, J.M., and Cole, J.R. (2007). Naive Bayesian classifier for rapid assignment of rRNA sequences into the new bacterial taxonomy. Appl. Environ. Microbiol. 73, 5261-5267.

Wang, Y., Rattner, A., Zhou, Y., Williams, J., Smallwood, P.M., and Nathans, J. (2012). Norrin/Frizzled4 signaling in retinal vascular development and blood brain barrier plasticity. Cell 151, 1332-1344. 


\section{STAR $\star M E T H O D S$}

\section{KEY RESOURCES TABLE}

\begin{tabular}{|c|c|c|}
\hline REAGENT or RESOURCE & SOURCE & IDENTIFIER \\
\hline \multicolumn{3}{|l|}{ Antibodies } \\
\hline rat anti-Ecadherin clone ECCD2 & Takara & Cat\#M108 \\
\hline mouse anti-ZO-1 & Invitrogen & Cat\#339100 RRID AB_2533147 \\
\hline mouse anti-Occludin & Invitrogen & Cat\#331500 RRID AB_2533101 \\
\hline mouse anti-Claudin- 5 & Invitrogen & Cat\#352500 RRID AB_2533200 \\
\hline $\begin{array}{l}\text { mouse anti-E-cadherin clone 36/E- } \\
\text { cadherin }\end{array}$ & BD Biosciences & Cat\#610181 \\
\hline rabbit anti-Claudin-3 & Sigma Aldrich & Cat\#SAB4500435-100UG \\
\hline rabbit anti-GBS & Thermo Scientific & Cat\#PA1-7250 RRID AB_561572 \\
\hline rabbit anti- $\beta$-catenin $\mathrm{H} 102$ & Santa Cruz & Cat\#sc.7199 \\
\hline rat anti-Ly6C ER-MP20 & Abcam & Cat\#ab54223 \\
\hline Alexa Fluor 488 anti-BrdU clone MoBU-1 & Life Technologies & Cat\#335130 RRID AB_2536434 \\
\hline $\begin{array}{l}\text { armenian hamster anti-PECAM1/CD31 } \\
\text { clone } 2 \mathrm{H} 8\end{array}$ & Millipore & Cat\#MAB1398Z \\
\hline mouse anti- $\beta$-actin clone AC- 15 & Sigma Aldrich & Cat\#A1978-200UL \\
\hline rat anti-PLVAP & Biorad & Cat\#MCA2639 \\
\hline Alexa Fluor 647 Wheat germ agglutinin & Invitrogen & Cat\#835738 \\
\hline Alexa Fluor 488 phalloïdin & Invitrogen & Cat\#A12379 \\
\hline Alexa Fluor 546 goat anti-mouse lgG1 & Invitrogen & Cat\#A21123 RRID AB_2535765 \\
\hline Alexa Fluor 647 goat anti-mouse IgG1 & Invitrogen & Cat\#A21240 RRID AB_2535809 \\
\hline Alexa Fluor 488 goat anti-rabbit & Invitrogen & Cat\#A11034 RRID AB_2576217 \\
\hline Alexa Fluor 546 goat anti-rabbit & Invitrogen & Cat\#A11035 RRID AB_2534093 \\
\hline Alexa Fluor 488 goat anti-rat & Invitrogen & Cat\#A11006 RRID AB_2534074 \\
\hline Alexa Fluor 546 goat anti-rat & Invitrogen & Cat\#A11081 RRID AB_2534125 \\
\hline Alexa Fluor 647 goat anti-rat & Invitrogen & Cat\#A21247 RRID AB_141778 \\
\hline Hoechst 33342 & Invitrogen & Cat\#H3570 \\
\hline $\begin{array}{l}\text { FITC conjugated lectin from } \\
\text { Lycopersicon esculentum }\end{array}$ & Sigma Aldrich & Cat\#L0401-1MG \\
\hline ECL anti-mouse IgG HRP & GE Healthcare & Cat\#NA931-100ul \\
\hline ECL anti-rabbit IgG HRP & GE Healthcare & Cat\#NA934-100ul \\
\hline \multicolumn{3}{|l|}{ Bacterial and virus strains } \\
\hline Streptococcus agalactiae CC17 BM110 & Claire Poyart & N/A \\
\hline \multicolumn{3}{|l|}{ Biological samples } \\
\hline Goat serum & Life Technologies & Cat\#16210-064 \\
\hline Fetal calf serum & Sigma Aldrich & Cat\#F9665-500ML \\
\hline \multicolumn{3}{|l|}{ Chemicals, peptides, and recombinant proteins } \\
\hline Todd-Hewitt Oxoid & Fisher Scientific & Cat\#11708962 \\
\hline Brain Heart Infusion (BHI) Difco & Fisher Scientific & Cat\#11708872 \\
\hline PBS Dulbecco's & Invitrogen & Cat\#11580456 \\
\hline $\begin{array}{l}\text { DMEM } 4.5 \mathrm{~g} / \text { L glucose Glutamax I } \\
\text { Pyruvate } \mathrm{Na} \text { GIBCO }\end{array}$ & Fisher Scientific & Cat\#12077549 \\
\hline Buprenorphine - Buprecare & Axience SAS & N/A \\
\hline Ketamine - Imalgene 1000 & Merial & $\mathrm{N} / \mathrm{A}$ \\
\hline xylazine chlorhydrate - Rompun & Bayer & N/A \\
\hline Vetadine savon/solution & Vetoquinol & N/A \\
\hline
\end{tabular}




\begin{tabular}{|c|c|c|}
\hline \multicolumn{3}{|l|}{ Continued } \\
\hline REAGENT or RESOURCE & SOURCE & IDENTIFIER \\
\hline Isofluorane - Iso Vet & Osalia & N/A \\
\hline Gentamicin $10 \mathrm{mg} / \mathrm{ml}$ & Sigma-Aldrich & Cat\#G1397-10ML \\
\hline Metformin & Sigma Aldrich & Cat\#PHR1084-500MG \\
\hline BrdU-labeling reagent solution & Life Technologies & Cat\#000103 \\
\hline FITC-Dextran 4kDa & Sigma-Aldrich & Cat\#46944-500MG-F \\
\hline Evans Blue & Sigma-Aldrich & Cat\#E2129-10G \\
\hline Paraformaldehyde $32 \%$ solution EM Grade & Interchim (EMS) & Cat\#15714-S \\
\hline Agarose ultrapure & Sigma Aldrich & Cat\#16500-500 \\
\hline Sucrose & Sigma Aldrich & Cat\#S9378-1KG \\
\hline $\begin{array}{l}\text { Tissue-Tek optimal cutting temperature } \\
\text { compound }\end{array}$ & Sakura Finetek France & Cat\#4583 \\
\hline Borate - Boric acid & Sigma Aldrich & Cat\#339067-100G \\
\hline Fluoromount G & Invitrogen & Cat\#00-4958-02 \\
\hline Triton $\mathrm{X}-100$ & Sigma Aldrich & Cat\#X100-100ML \\
\hline Trizol & Ambion & Cat\#15596026 \\
\hline chloroform-isoamyl alcohol & Sigma-Aldrich & Cat\#25666-500ML \\
\hline RIPA Buffer & Sigma Aldrich & Cat\#R0278-50ML \\
\hline benzonase nuclease & Sigma Aldrich & Cat\#E1014-25KU \\
\hline \multicolumn{3}{|l|}{ Critical commercial assays } \\
\hline $\begin{array}{l}\text { High capacity cDNA reverse transcription } \\
\text { kit }\end{array}$ & Thermo Fisher Scientific & Cat\#4368814 \\
\hline Taqman Universal master mix II & Thermo Fisher Scientific & Cat\#4426710 \\
\hline BCA Protein assay & Pierce & Cat\#23225 \\
\hline DNeasy PowerSoil Kit & QIAGEN & Cat\#12888-100 \\
\hline \multicolumn{3}{|l|}{ Experimental models: Organisms/strains } \\
\hline Conventional C57BL/6JRj mice & Janvier Laboratories & Cat\#SC-CJ-6S-F Cat\#SC-CJ-6S-M \\
\hline GF C57BL/6JRj mice & $\begin{array}{l}\text { Institut Pasteur, gnotobiology } \\
\text { and animal facility platforms }\end{array}$ & $\mathrm{N} / \mathrm{A}$ \\
\hline \multicolumn{3}{|l|}{ Oligonucleotides } \\
\hline Taqman Gene Expression assay (FAM) & Life Technologies & Cat\#4331182 \\
\hline \multicolumn{3}{|l|}{ Software and algorithms } \\
\hline Fiji - ImageJ & ImageJ & https://imagej.net/Fiji \\
\hline $\mathrm{R}$ & $R$ foundation & https://www.r-project.org/ \\
\hline Arivis & Arivis & https://www.arivis.com/ \\
\hline Prism 8.0 & GraphPad & $\begin{array}{l}\text { https://www.graphpad.com/ } \\
\text { scientific-software/prism/ }\end{array}$ \\
\hline \multicolumn{3}{|l|}{ Other } \\
\hline BD GasPak EZ & Becton Dickinson and Company & Cat\#11777194 \\
\hline
\end{tabular}

\section{RESOURCE AVAILABILITY}

\section{Lead contact}

Further information and requests for resources and reagents should be directed to and will be fulfilled by the lead contact, Marc Lecuit (marc.lecuit@pasteur.fr).

Materials availability

This study did not generate new unique reagents. 
Data and code availability

The published article includes all datasets generated or analyzed during this study, with exception of microbiota sequences that have not been deposited in a public repository but are available from the corresponding author on request.

\section{EXPERIMENTAL MODEL AND SUBJECT DETAILS}

\section{Animals}

Conventional 6 to 10 weeks-old male and female adult specific pathogen free and GF C57BL/6JRj mice were provided by Janvier Laboratories and breed and housed in the same animal facility at least two weeks before being handled for experiments to avoid a bias coming from microbiota differences and mixed with the other animals in the same cage if possible. Six to 10 weeks-old male and female adult GF C57BL/6JRj were breed and housed in dedicated isolators. We included in this study male and female embryonic E18-20, post-natal P3, P4, P5, P6, P9, P10 and P15 mice obtained after breeding of conventional and GF C57BL/6JRj in individually ventilated cages or isolators. All the procedures were in agreement with the guidelines of the European Commission for the handling of laboratory animals, directive 86/609/EEC. They were approved by the ethical committees No. 59 by the CETEA/ CEEA No. 89 under the number 2013-0099, 01454.02 and APAFIS \#19384-2019022112019218.

\section{Bacteria}

We used GBS CC17 BM110 isolate kindly provided by Claire Poyart.

\section{METHOD DETAILS}

\section{Mice treatments and infections}

For infections, GBS CC17 BM110 fresh colonies were diluted in Todd-Hewitt medium to reach stationary phase. We used a concentration of GBS per gram of mice to avoid artificial overloading of neonatal mice that would show even higher bacterial burden in these mice. We used a higher inoculum for oral inoculations compared to i.p/intravenous (IV) inoculations: after oral inoculation, intestinal barrier filtrate bacteria and limit dissemination to systemic organs and brain as compared to direct inoculation in systemic circulation. For oral inoculations, $5.10^{8}$ GBS per gram were routinely injected intragastrically in adults or in the lower part of the pharynx of neonates after resuspension in $10 \mu \mathrm{L}$ of PBS (see Figures $1 \mathrm{~A}, 1 \mathrm{E}-1 \mathrm{G}, 2 \mathrm{~B}, \mathrm{~S} 3 \mathrm{~A}, 7 \mathrm{C}$, and S8D). For colonization resistance assay, we orally-inoculated $10^{7}$ per gram to keep mice healthy for 5 days (see Figure $2 \mathrm{~A}$ ). We performed IV inoculations in experiments aiming to compare adult mice. Since IV inoculations in neonates may lead to artifactual infection of the brain near the cheek vein inoculation site, i.p inoculations were performed when comparing neonates and adults. I.p. and IV inoculation were performed with $2,5.10^{5} \mathrm{GBS}$ per gram resuspended in $10 \mu \mathrm{L}$ of PBS (see Figures 4D and 4E). For i.p. infections of P15 conventional and conventionalized P15 conv+mic (see Figure 4F), we increased the inoculum of GBS by $10\left(2,5.10^{6}\right.$ GBS per gram) to better analyze the effect of mature microbiota on susceptibility to GBS infection. To build our mathematical model (see Figures 5C, 5D, S5F, and S5G), we also performed short-term i.p. inoculations to analyze early events of GBS crossing through BBB interfaces and study BBB interfaces permissiveness limiting GBS multiplication and elimination by the immune system over the time. However, to maximize odds of GBS crossing in this short time period and analyze events, we increased the inoculum to $10^{6}$ per gram. We also included embryonic E18-20 mice to better study the role of the age on BBB interfaces permissiveness without the potential bias that the installing microbiota, and the subsequent difference on ability of P5 conventional versus P5 GF to control bacteremia (Deshmukh et al., 2014), may induce on invasion of the BBB interfaces. I.p. injection of E18-20 embryos was realized after laparotomy of pregnant mice: 30 min after injection of the buprenorphine $(0.1 \mathrm{mg} / \mathrm{kg}$ body weight), deep anesthesia was induced with a mix of ketamine (100 mg/kg body weight; Imalgene 1000; Merial), and xylazine chlorhydrate (10 mg/kg body weight; Rompun; Bayer). Skin was cleaned with Vetadine, a laparotomy was performed, the uterus was exposed and opened and embryos were extracted from the amniotic sac without sectioning connections to placenta. Mother and embryos were sacrificed $1 \mathrm{~h}$ after inoculation and in the meantime, PBS was injected subcutaneously to the mother to prevent dehydration. We also performed $1 \mathrm{~h}$ i.p. inoculations with $10^{6}$ per gram to analyze by microscopy the repartition of GBS in the brain (see Figures 5A, 5B, and S5E) and compare GBS count between $1 \mathrm{~h}$ and $16 \mathrm{~h}$ post-i.p. inoculation (see Figure S6A and associated microscopy analysis Figure S6B).

To monitor bacterial burden, gut luminal content, blood, and organs were removed from infected killed animals and homogenized. When CP and BMB were dissected from brain parenchyma, mice blood had been first removed after intracardiac perfusion with PBS. Before homogenization, small intestine and colon tissues were rinsed twice with DMEM and incubated for $2 \mathrm{~h}$ at room temperature in DMEM supplemented with gentamicin ( $100 \mathrm{mg} / \mathrm{ml}$; Sigma-Aldrich). Serial dilutions of cell suspensions in PBS were plated on brainheart infusion agar plates. After $24 \mathrm{~h}$ of incubation at $37^{\circ} \mathrm{C}$, GBS colony forming units were enumerated.

To conventionalize GF adult and conventional neonatal mice, the whole gut luminal content from conventional adult mice was orally-inoculated to mice: once two weeks before infection of GF adult mice; at P3, P6 and P9 before infection of conventional P15 mice (see Figures 1F, 1G, S1C, 4E, and 4F).

Conventional P4 mice were orally inoculated with $300 \mu \mathrm{g} / \mathrm{g} /$ day of metformin (Sigma Aldrich), for 2 days (see Figures $7 \mathrm{~A}-7 \mathrm{C}$ and S8B-S8D). 
For BrdU assays, $7.5 \mu \mathrm{l}$ per gram of BrdU-labeling reagent solution (Life Technologies) were i.p.-injected $16 \mathrm{~h}$ before sacrifice (see Figures S4C, S4D, and S7G).

Intestinal and CP permeability assays

Permeability of the IB was assessed after oral inoculation of 0,5 mg per gram of FITC-Dextran $4 \mathrm{kDa}$ (Sigma-Aldrich) and mice blood was sampled $4 \mathrm{~h}$ after the injection. FITC-Dextran $4 \mathrm{kDa}$ concentration was measured at $488 \mathrm{~nm}$ using a spectrophotometer (see Figure 2C).

Permeability of the CP barrier was analyzed by sampling CSF from mice that were i.p.-inoculated $2 \mathrm{~h}$ before with $0,2 \mathrm{mg}$ per gram of Evans Blue (Sigma-Aldrich). For CSF sampling in adult mice, animals were first anesthetized with $100 \mathrm{mg} / \mathrm{kg} \mathrm{ketamine} \mathrm{and} 10 \mathrm{mg} / \mathrm{kg}$ xylazine, diluted in sterile saline. Mice were then positioned in a stereotaxic frame, and craniotomies were performed. CSF was sampled from the lateral ventricle bilaterally $(-0.2 \mathrm{~mm}$ Antero-Posterior and \pm 1 Medio-Lateral from bregma, and $-1.9 \mathrm{~mm}$ Dorso-Ventral from brain surface) with a glass micropipette connected to a Nanoject II microinjector (extraction speed $20 \mathrm{nl} / \mathrm{sec}$ ). For CSF sampling in neonatal mice, pups at P5 were anesthetized with isofluorane $\left(3.5 \% ; 372 \mathrm{~mL} \mathrm{~min}^{-1}\right)$ and positioned in a stereotaxic frame using a homemade cast. A glass pipette was used to collect CSF by puncture of the Cisterna Magna through the dura mater. Following a noticeable change in resistance to the capillary tube insertion into the cisterna magna, the CSF flows into the capillary tube. The capillary tube was carefully removed and connected to a $20 \mathrm{~mL}$ syringe through a polyethylene tubing that has a $1 \mathrm{~mm}$ internal diameter. Both for adult and neonate mice the CSF was collected and Evans blue concentration was measured at $620 \mathrm{~nm}$ using a spectrophotometer (see Figure 5E).

\section{Immunofluorescence}

Tissues were labeled as previously described (Disson et al., 2009). Before vibratome sectioning, dissected intestines, colons, and brains were fixed in $4 \%$ paraformaldehyde overnight. Tissues were then washed in PBS and then either embedded in agarose for slices using a vibratome or immerged in $30 \%$ sucrose overnight and embedded in optimal cutting temperature compound for cryostat sectioning. For brain sectioning by cryostat, dissected brains were embedded in optimal cutting temperature compound unfixed but slices were incubated $10 \mathrm{~min}$ in $100 \%$ ethanol at $-20^{\circ} \mathrm{C}$ and rehydrated 10 min in water and 10 min in PBS before staining. Slices of $40-\mu \mathrm{m}$-thick (brains) or $150-\mu \mathrm{m}$-thick (intestines, colons) were performed with vibratome whether slices of 10 - $\mu \mathrm{m}$-thick (intestines, colons) or $25-\mu \mathrm{m}$-thick (brains) were realized with cryostat.

For BrdU staining, samples were incubated sequentially in $\mathrm{HCl} 1 \mathrm{~N}$ for 10 min at $4^{\circ} \mathrm{C}, \mathrm{HCl} 2 \mathrm{~N}$ for 10 min at room temperature, $\mathrm{HCl} 2$ $\mathrm{N}$ for $20 \mathrm{~min}$ at $37^{\circ} \mathrm{C}$, buffered with borate buffer $(0.1 \mathrm{M})$ for $12 \mathrm{~min}$ at room temperature, and washed in PBS, before staining.

For staining, slices tissues were incubated for $2 \mathrm{~h}$ in blocking-permeabilization solution (10\%/4 \% goat serum for brain/intestine and colon, respectively, $4 \%$ fetal calf serum and $0.4 \%$ Triton X-100 in PBS). Tissues were then labeled with the appropriate primary antibodies overnight at $4^{\circ} \mathrm{C}$ in mild blocking conditions, washed in PBS, then incubated with secondary antibodies for $2 \mathrm{~h}$ at room temperature. Tissues were washed in PBS and then mounted on glass slides under coverslips in mounting medium (Fluoromount G; Beckman Coulter). Dissected whole CP were stained on ice in $4 \%$ goat serum, $4 \%$ fetal calf serum and $0.06 \%$ Triton X-100 in PBS $+/$ + before fixation in $4 \%$ paraformaldehyde for $20 \mathrm{~min}$, washing in PBS and tissue mounting. The slides are let in obscurity overnight at room temperature before observation under a Zeiss LSM700 and a Zeiss LSM710 microscope equipped with Airyscan super-resolution module.

We used the following primary antibodies: rat anti-E-cadherin clone ECCD-2 (Takara), mouse anti-ZO-1, mouse anti-Occludin, mouse anti-Claudin-5 are from Invitrogen, mouse anti-E-cadherin (BD Biosciences), rabbit anti-Claudin-3 (Sigma Aldrich), rabbit anti-GBS (Thermo Scientific), rabbit anti- $\beta$-catenin H102 (Santa Cruz), rat anti-Ly6C (ER-MP20 Abcam), Armenian hamster anti-PECAM1/CD31 clone 2H8 (Millipore), rat anti-PLVAP (Biorad). The flowing secondary antibodies were used: Alexa Fluor anti-BrdU clone MoBU-1 (Life Technologies), Alexa Fluor 647 Wheat germ agglutinin, Alexa Fluor 488 phalloïdin, Alexa Fluor 546 and Alexa Fluor 647 goat anti-mouse IgG1, Alexa Fluor 488 and Alexa Fluor 546 goat anti-rabbit, Alexa Fluor 488, Alexa Fluor 546 and Alexa Fluor 647 goat anti-rat and Hoechst 33342 that all are from Invitrogen as well as FITC conjugated lectin from Lycopersicon esculentum (Sigma Aldrich).

\section{Mathematical modeling}

To study the BBB function of the three BBB interfaces, we developed a mathematical model of bacterial dissemination. Liver, spleen, $\mathrm{CP}, \mathrm{BMB}$ and parenchyma are separated compartments connected by the bloodstream, treated as a sixth compartment. The model tracked the number of GBS through time in six compartments and accounted for bacterial clearance in the blood as well as for the flow between different compartments: flow back and forth of bacteria from the blood to the liver and the spleen that could influence the concentration of GBS upstream the three BBB interfaces; the flow of GBS from blood to CP, BMB and brain microvessels (parenchyma); bacterial burden in the BMB could reflect crossing events through the meningeal blood vessels but also crossing events that occurred through CP followed by transportation to the subarachnoid space of the BMB via the CSF flow; bacteria in the BMB could also directly reach the parenchyma. Given that the animals were sacrificed just $1 \mathrm{~h}$ after i.p. inoculation, we neglected bacterial replication. The overall clearance occurring in between the inoculation and the sacrifice is represented by the death parameter. 
The system of differential equations describing the model are given by:

$$
\begin{gathered}
\frac{d B}{d t}=-\left(\mu_{B I}+k_{B I-L i}+k_{B I-S P}+k_{B I-C P}+k_{B I-B M B}+k_{B I-P}\right) B(t)+k_{L i-B I} L(t)+k_{S P-B I} S(t) \\
\frac{d L}{d t}=k_{B I-L i} B(t)-k_{L i-B I} L(t) \\
\frac{d S}{d t}=k_{B I-S P} B(t)-k_{S P-B I} S(t) \\
\frac{d C}{d t}=k_{B I-C P} B(t)-k_{C P-B M B} C(t) \\
\frac{d M}{d t}=k_{B I-B M B} B(t)+k_{C P-B M B} C(t)-k_{B M B-P} M(t) \\
\frac{d P}{d t}=k_{B I-P} B(t)+k_{B M B-P} M(t)
\end{gathered}
$$

where the number of bacteria at time $t$ in the blood, liver, spleen, CP, BMB and parenchyma are denoted respectively as $B(t), L(t), S(t)$, $C P(t), B M B(t)$, and $P(t)$. The parameter $\mu_{\mathrm{BI}}$ represents the clearance rate in the blood while the other parameters capture flux rates: for example, $\mathrm{k}_{\mathrm{BI}-\mathrm{Li}}$ represents the flux rate from the blood to the liver. All parameters were all assumed to be time-independent and a separate set of parameters was used for each of the five mouse populations considered (conventional E18-20, P5 and adult and GF P5 and adult).

The system of differential equations was solved using Runge-Kutta's method (Press et al., 1992) with a time step of $0.1 \mathrm{~h}$ and was initialized assuming that the number of bacteria in all compartments - except for the blood, for which we used the nominal inoculum was zero.

The model was calibrated on GBS enumerated in the blood, liver, spleen, and in the three BBB interfaces assuming a lognormal measurement process with a standard deviation that was jointly estimated along with the other parameters.

Parameters were estimated using Markov Chain Monte Carlo sampling (Gilks et al., 1996) using flat, non-informative, priors. Chain convergence was assessed by visual inspection of the trace plots.

\section{Quantitative real-time PCR (qRT-PCR)}

RNA isolation was performed after lysis of the intestinal, colon and CP tissues in Trizol ${ }^{\circ}$ reagent (Ambion) using Precellys Lysing Kit CKMix (Ozyme) and extraction of RNA with chloroform-isoamyl alcohol mixture (Sigma-Aldrich). mRNA (500 ng) was transcribed into cDNA using High capacity cDNA reverse transcription kit (Thermo Fisher Scientific). All procedures were performed according to the manufacturers' protocols. We performed qPCR using Taqman Gene Expression assay (FAM) (Life Technologies) on a StepOnePlus Real-Time PCR system (Applied Biosystems). We normalized Ct values to the mean Ct values obtained for the housekeeping genes Gapdh, Hprt, Rp/37a and Ppia.

\section{Western blotting}

CP were prepared with RIPA buffer (Sigma Aldrich) and boiled for 5 min in loading buffer with benzonase nuclease (Sigma Aldrich). Equal amounts of lysates protein were loaded and separated on either a $10 \%$ or a $14 \%$ SDS-polyacrylamide gel electrophoresis gel and transferred onto a PVDF membrane before analysis. The protein concentration was assayed from the resulting supernatants by BCA'method (Pierce). Samples were immunoblotted with the appropriated primary antibodies: mouse anti-E-cadherin (BD Biosciences), rabbit anti- $\beta$-catenin (Santa Cruz) and mouse anti- $\beta$-actin clone AC-15 (Sigma Aldrich) followed by anti-mouse and anti-rabbit IgG HRP secondary antibodies (GE Healthcare). Protein levels were quantified by measuring the intensity of the bands by densitometry using Fiji Software (see Figure S7F).

\section{Microbiota analysis}

Quantification of commensals from gut luminal content (see Figure 1B) of conventional P3, P6, P9, P15, P24 and adult was performed quickly after animal sacrifice by serial dilution in PBS and plating on Columbia agar. Plates were incubated at $37^{\circ} \mathrm{C}$ for 4 days both in aerobic and anaerobic conditions using BD GasPak EZ (Becton Dickinson and Company). 
For metagenomic analysis of the microbiota composition (see Figures 1C, 1D, S1A, S1B, S1D, and S1E), DNAs from gut lumen contents of conventional P5, P10, P15 and adult as well as conventionalized P15 (see Figure S1C) and GF adult was extracted using DNeasy PowerSoil Kit (QIAGEN) accordingly to the manufacturer's instructions. The V4 region has been amplified and sequenced with the primers CCTACGGGNGGCWGCAG and GACTACNVGGGTWTCTAATCC using the Illumina MiSeq workflow at the biomics platform at the Institut Pasteur, Paris. Analysis have been performed with MICCA (Albanese et al., 2015), using the RDP classifier (Albanese et al., 2015), which uses VSEARCH to match sequences in the reference database (Rognes et al., 2016). Forward and reverse reads were merged with a minimum overlap of $100 \mathrm{bp}$ and 30 maximum allowed mismatches. Forward and reverse primers were removed and reads were trimmed to 400 nucleotides using the MICCA workflow. Reads with an expected error rate above 0.75 $\%$ were excluded. Reads were grouped in operational taxonomic units (OTUs) with the MICCA function 'otu' and the 'denovo_unoise' option (Edgar, 2015). Chimeric sequences were removed. OTUs were classified with RDP (Wang et al., 2007), which uses VSEARCH to match sequences in the reference database (Rognes et al., 2016). OTU relative abundance has been expressed as a fraction of a given OTU out of all OTUs in a rarefied sample. Alpha diversity has been calculated in samples by the Shannon index (which considers the evenness of abundance of present species) (Hill, 1973). Beta diversity between samples has been expressed as PCoA/MDS of Bray-Curtis dissimilarities (Bray and Curtis, 1957). Statistical analysis and visualization were performed using R software and the phyloseq library (McMurdie and Holmes, 2015). PERMANOVA and homogeneity between microbiome groups were calculated with adonis and betadispers from the vegan library (Oksanen et al., 2012). Comparison of variance within groups between all groups was performed using Permutation test for homogeneity of multivariate dispersions. Significantly different OTUs between samples were identified using DESeq2 (Love et al., 2014).

\section{QUANTIFICATION AND STATISTICAL ANALYSIS}

\section{Image analysis}

Each image is representative for at least four analyzed animals per condition/age, both male and female, for which several images have been taken and processed. To normalize variation in P5 versus adult mice tissue architecture, we imaged the entire sectioned tissue and/or villi and analyzed intensity signals either at the same depth in both fields or at the same depth in imaged villi. Image analysis was performed using Fiji software.

Repartition of E-cadherin, Occludin and Claudin-3 signals along intestinal epithelial junctions was realized after normalization of the junction length, based on draw intestinal epithelial junctions on six different images realized from tissues of three different mice. The results in Figures 2E-2G and S3D-S3F showed the analysis of the junctions of one image representative of the studied groups: 85 draw junctions in conventional P5 and 60 in conventional adult (see Figure 2D) and 40 draw junctions in conventional adult and 39 in GF adult (see Figure S3C). Signal distribution was compared at 0.2 and 0.5 of normalized length (regions with signal peak in conventional and GF adult and conventional P5) using a two-tailed non-parametric Mood test with the null hypothesis that two compared samples come from populations with the same median with a significance level of alpha $=0.025+0.025=0.05$. The significance levels are derived as followed: ${ }^{*}=(p<0.05)$ and $(p>=0.01) ;{ }^{* \star}=(p<0.01)$ and $(p>=0.001) ;{ }^{* \star *}=p<0.001$.

Cellular membranous and cytoplasmic signal intensity for $\beta$-catenin and E-cadherin in intestinal epithelial cells was analyzed along drawn white line on six different images realized from tissues of three different mice: see Figures $3 \mathrm{~A}$ and $\mathrm{S} 4 \mathrm{~A}$ and see Figure $3 \mathrm{~B}$ showing signal intensity profile of a representative image of the P5 conventional versus adult conventional groups. Each point of Figures $3 C$ and 3D and Figure S4B represent the values for P5 conventional $(n=32)$ versus adult conventional $(n=32)$ and for adult conventional $(n=39)$ versus adult GF $(n=29)$ cytoplasmic/membranous intensity signals, obtained from these drawn white line on six different images.

$\mathrm{BrdU}^{+}$intestinal epithelial cells were enumerated in six different images of conventional P5 and adult intestine and are represented as a percentage over the number of counted epithelial cells nuclei (see Figure S4D).

PLVAP and CD31 signals quantification (see Figure 4B) was performed drawing the circumference of CD $31^{+}$area in each villus (see light gray dots on Figure 4A) of conventional P5 ( $n=62$ villi) and adult ( $n=62$ villi) as well as GF adult ( $n=56$ villi). PLVAP and Claudin-5 signals quantification (see Figures S5B and S5C) was realized drawing the external face of the villi endothelium (see light gray dots on Figure S5A) of conventional P5 $(n=26)$ and adult $(n=26)$ and GF P5 $(n=17)$ and adult $(n=26)$.

$\mathrm{CP}$ cell area, junction length per $\mu \mathrm{m}^{2}$ of $\mathrm{CP}$ cell area (see Figures $6 \mathrm{~B}$ and $\mathrm{S} 7 \mathrm{~B}$ ) were quantified drawing the conventional P5 and adult CP cell circumference on Figure S7A ( $n=100$ cells in both groups). Junction length of CP per $1,000 \mu \mathrm{m}^{2}$ of tissue (see Figure S7C) was measured on six different area of $1,000 \mu \mathrm{m}^{2}$ in Figure S7A in both conventional P5 and adult. Repartition of ZO-1 signal intensity along CP junctions (see Figure S7D) was analyzed drawing junctions between two cells and from intersections of three or four cells to another from sections in Figure $6 \mathrm{~A}$.

Three-dimensional reconstructions were performed using Arivis software using planal clipping.

Data and statistical analysis

Data are represented with median and interquartile range.

Exact values of $n$ samples are specified in respective figure legends. Number of samples may vary between the different groups of an experiment: to analyze the respective contribution of the age per se and the microbiota in neonatal susceptibility to GBS meningitis, we emphasized our analysis on three main groups: conventional P5 and adult mice as well as GF adult mice and size of 
conventional P10 and P15 groups may be lower as they are only used to illustrate the age-dependent decrease of susceptibility to GBS infection. In addition, in experiments showing equivalent distribution between two groups (see conventional and GF P5 in Figure 2C for example), we did not use more P5 GF mice for ethical reason because it would require too many animals to see a minor difference, if this latest exists. Finally, in experiments showing significant difference between groups with a low number of samples (see adult GF group in Figure 4D for example), for ethical reason, we did not include more animals.

Statistical analyses were performed with Prism 8.0 software. Used statistical tests are specified at the end of Figure legends. Nonparametric Mann-Whitney tests were routinely used for statistical analysis implicating only two groups. Statistical analysis of more than two groups were performed using One-way ANOVA followed by Holm-Sidak's multiple comparisons, Brown-Forsythe and Welch ANOVA followed by Dunnett's T3 multiple comparisons when groups repartition harbored different standard deviation (qRTPCR data, concentration of Evans blue in CSF, PLVAP, CD31 and Claudin-5 signals quantification) or non-parametric Kruskal-Wallis ANOVA followed by Dunn's multiple comparisons in cases of GBS enumeration within blood or organs, when data do not follow a normal distribution. For analysis of correlation between GBS count in colon tissue and lumen, a Spearman non-parametric correlation was used. Statistical significance is expressed as follows: ${ }^{\star}, p<0.05 ;{ }^{\star \star}, p<0.01 ;{ }^{\star \star \star}, p<0.001 ;{ }^{* \star \star \star}, p<$ 0.0001 . Two-tailed non-parametric Mood test was performed for analysis of E-cadherin, Claudin-3 and Occludin signals repartition along intestinal epithelial junctions (see above) with the null hypothesis that two compared samples come from populations with the same median with a significance level of alpha $=0.025+0.025=0.05$. The significance levels are derived as followed: ${ }^{*}=(p<0.05)$ and $(p>=0.01) ;{ }^{* *}=(p<0.01)$ and $(p>=0.001) ;{ }^{* *}=p<0.001$. PermANOVA statistical test was performed for analysis of the microbiota composition using $\mathrm{R}$ software (see above). 